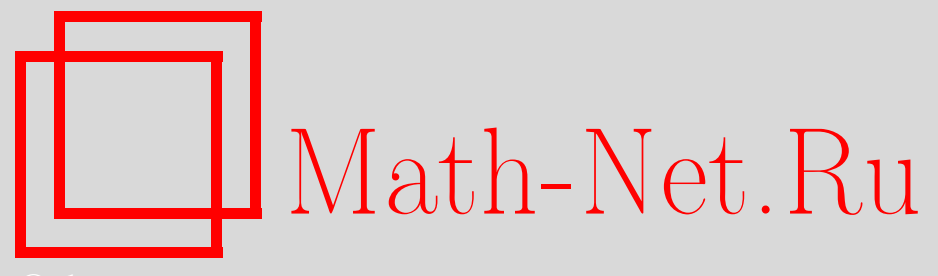

В. А. Ведерников, М. М. Сорокина, $\Omega$-расслоенные формации и классы Фиттинга конечных групп, Дискрет. матем., 2001, том 13, выпуск 3, 125-144

DOI: https://doi.org/10.4213/dm299

Использование Общероссийского математического портала Math-Net.Ru подразумевает, что вы прочитали и согласны с пользовательским соглашением http://www . mathnet.ru/rus/agreement

Параметры загрузки:

IP : 54.197 .130 .99

26 апреля 2023 г., 10:49:28

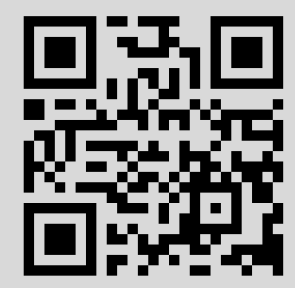




\title{
Л-расслоенные формации и классы Фиттинга конечных групп
}

\author{
(C) 2001 г. В. А. Ведерников, М. М. Сорокина
}

Предложен новый функциональный подход к исследованию классов групп, позволивший на языке функций описать все формации и классы Фиттинга конечных групп. Построены $\Omega$-расслоенные формации $\Omega F(f, \varphi)$ и $\Omega$-расслоенные классы Фиттинга $\Omega F(f, \varphi)$ со спутником $f$ и направлением $\varphi$. Каждому спутнику $f$ соответствует бесконечное множество различных направлений $\varphi$. Одно из направлений приводит к ранее рассмотренным $\Omega$-композиционным формациям. На этом пути получены $\Omega$-канонические и $\Omega$-свободные формации и классы Фиттинга. При фиксированном направлении $\varphi$ получено строение минимального спутника $f$.

\section{1. Введение}

Понятие формации было введено в 1963 году Гашюцем в [1], и уже в этой работе функциональные методы нашли применение в построении формаций. А именно, Гашюцем были введены локальные формации, занимающие центральное место в теории формаций. Дальнейшее развитие функциональный подход к изучению формаций нашел в работе Л. А. Шеметкова [2], в которой были определены ступенчатые, примарно постоянные, однородные и композиционные формации. Отметим, что композиционные формации были построены также Бэром (см. [3]), а Хартли в [4] определил локальные классы Фиттинга. В последние годы Л. А. Шеметковым и А. Н. Скибой интенсивно исследуются частично локальные ( $\omega$-локальные) формации и классы Фиттинга (см., например, $[5,6])$. В $[7,8]$ независимо определены и исследованы $\Omega$-композиционные формации с помощью $\Omega$-композиционного спутника $f$, где $\Omega$ - непустой подкласс класса $\mathfrak{I}$ всех простых групп. Идея построения новых видов формаций и классов Фиттинга с помощью $\Omega$-спутников привела к необходимости рассмотрения $\Omega$-спутников различных направлений, причем направление $\Omega$-спутника $f$ определяется как отображение класса $\mathfrak{I}$ в множество всех непустых формаций Фиттинга. Ясно, что такие направления образуют бесконечное множество, и значит, для фиксированного класса $\Omega$ можно построить бесконечное множество новых видов формаций ( $\Omega$-расслоенных формаций).

Все рассматриваемые группы предполагаются конечными. Приведем некоторые обозначения и определения, другие обозначения и определения, встречающиеся в статье, можно найти в книгах $[9,10]$. Пусть $\mathfrak{X}$ - непустое множество групп. Тогда $(\mathfrak{X ) ~ о б о з н а ч а е т ~ к л а с с ~ г р у п п , ~ п о р о ж д е н н ы и ̆ ~} \mathfrak{X}$; в частности, $(G)$ - класс всех групп, 
изоморфных группе $G ; K(G)$ - класс всех простых групп, изоморфных композиционным факторам группы $G ; K(\mathfrak{X})$ - объединение классов $K(G)$ для всех $G \in \mathfrak{X}$. Пусть $\mathfrak{I}$ - класс всех простых конечных групп, $\Omega$ - непустой подкласс класса $\mathfrak{I}$. Если $K(G) \subseteq \Omega$, то $G$ называется $\Omega$-группой. Через $\mathfrak{G}_{\Omega}$ обозначают множество всех $\Omega$-групп [7] и полагают что $1 \in \mathfrak{G}_{\Omega}$. Пусть $A \in \mathfrak{I}$. Тогда $\mathfrak{G}_{A}=\mathfrak{G}_{(A)}, A^{\prime}=\mathfrak{I} \backslash(A)$. Главный фактор $H / K$ группы $G$ называется главным $A$-фактором, если $K(H / K)=(A)$. Через $F_{A}(G)$ обозначают пересечение централизаторов всех главных $A$-факторов группы $G$. Если в $G$ нет главных $A$-факторов, то полагают $F_{A}(G)=G[11]$. Запись $M \triangleleft G$ означает, что $M$ является нормальной подгруппой группы $G$. Пусть $\mathfrak{F}$ - формация (класс Фиттинга). Тогда $G^{\mathfrak{F}}-\mathfrak{F}$-корадикал $\left(G_{\mathfrak{F}}-\mathfrak{F}\right.$-радикал) группы $G$. Будем полагать

$$
O_{\Omega}(G)=G_{\mathfrak{G} \Omega}, \quad O^{\Omega}(G)=G^{\mathfrak{B} \Omega}, \quad O_{\Omega^{\prime}, \Omega}=G_{\mathfrak{G} \Omega^{\prime} \mathfrak{B} \Omega}, \quad O^{\Omega, \Omega^{\prime}}=G^{\mathfrak{G} \Omega \mathfrak{G} \Omega^{\prime}} .
$$

Через $\mathfrak{F H}$ будем обозначать гашюцево произведение формаций $\mathfrak{F}$ и $\mathfrak{H}$, то есть класс всех таких групп $G$, для которых $G^{\mathfrak{H}} \in \mathfrak{F}$.

\section{2. $\Omega$-расслоенные формации}

Определение 1. Функцию $f: \Omega \cup\{\Omega\}^{\prime} \rightarrow\{$ формации групп $\}$, принимающую одинаковые значения на изоморфных группах из $\Omega$, назовем $\Omega$-формационной функцией или, коротко, $\Omega F$-функцией. Функцию $g: \mathfrak{I} \rightarrow\{$ формации групп $\}$, принимающую одинаковые значения на изоморфных группах из $\mathfrak{I}$, назовем формационной функцией или, коротко, $F$-функцией. Функцию $\varphi: \mathfrak{I} \rightarrow\{$ непустые формации Фиттинга $\}$, принимающую одинаковые значения на изоморфных группах из I, назовем формационно-радикальной функцией или, коротко, $F R$-функцией.

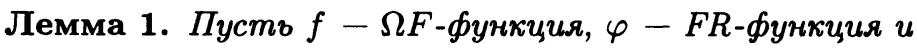

$$
\begin{aligned}
\mathfrak{F} & =\Omega F(f, \varphi) \\
& =\left\{G \in \mathfrak{H} \mid G / O_{\Omega}(G) \in f\left(\Omega^{\prime}\right) u G / G_{\varphi(A)} \in f(A) \text { для всех } A \in \Omega \cap K(G)\right\} .
\end{aligned}
$$

Тогда $\mathfrak{F}$ является формацией.

Доказательство. Пусть $g \in \mathfrak{F}$ и $N \triangleleft G$. Так как

$$
N O_{\Omega}(G) / N \cong O_{\Omega}(G) /\left(N \cap O_{\Omega}(G / N)\right) \in \mathfrak{G}_{\Omega},
$$

то $N O_{\Omega}(G) / N \subseteq O_{\Omega}(G / N)=R / N$. Поэтому

$$
(G / N) / O_{\Omega}(G / N) \cong G / R \cong\left(G / O_{\Omega}(G)\right) /\left(R /\left(R / O_{\Omega}(G)\right) \in f\left(\Omega^{\prime}\right) .\right.
$$

Поскольку для любого $A \in \Omega \cap K(G / N)$ верно $N G_{\varphi(A)} / N \cong G_{\varphi(A)} / N \cap G_{\varphi(A)} \in \varphi(A)$, то $N G_{\varphi(A)} / N \subseteq(G / N)_{\varphi(A)}$ и

$$
(G / N) /(G / N)_{\varphi(A)} \cong G / T \cong\left(G / G_{\varphi(A)}\right) /\left(T / G_{\varphi(A)}\right) \in f(A)
$$

для всех $A \in \Omega \cap K(G / N)$. Следовательно, $G / N \in \mathfrak{F}$.

Пусть $G / N_{i} \in \mathfrak{H}, i=1,2$. Покажем, что $G / D \in \mathfrak{F}$, где $D=N_{1} \cap N_{2}$. Ясно, что $G / D$ изоморфно подпрямому произведению групп $G / N_{1}$ и $G / N_{2}$. Поэтому существует такая группа $T$, что $T=K \times L, H$ - подпрямое произведение 
групп $K$ и $L$, причем $K \cong G / N_{1}, L \cong G / N_{2}$ и $H \cong G / D$. Так как $\mathfrak{G}_{\Omega}$ является формацией Фиттинга, по лемме 8 из [7] $O_{\Omega}(H)=H \cap O_{\Omega}(T)$. Далее, $O_{\Omega}(T)=$ $O_{\Omega}(K) \times O_{\Omega}(L)$ и $O_{\Omega}(T) H$ является подпрямым произведением групп $K$ и $L$. Тогда по лемме 2 из [12] $H O_{\Omega}(T) / O_{\Omega}(T)$ является подпрямым произведением групп $K O_{\Omega}(T) / O_{\Omega}(T) \cong K / O_{\Omega}(K)$ и $L O_{\Omega}(T) / O_{\Omega}(T) \cong L / O_{\Omega}(L)$. Так как $K / O_{\Omega}(K) \cong$ $\left(G / N_{1}\right) / O_{\Omega}\left(G / N_{1}\right) \in f\left(\Omega^{\prime}\right)$ и $L / O_{\Omega}(L) \in f\left(\Omega^{\prime}\right)$, то $H O_{\Omega}(T) / O_{\Omega}(T) \cong H / O_{\Omega}(H) \in$ $f\left(\Omega^{\prime}\right)$, и значит, $(G / D) / O_{\Omega}(G / D) \in f\left(\Omega^{\prime}\right)$. Поскольку $\varphi(A)$ - формация Фиттинга, аналогично получим, что $(G / D) /(G / D)_{\varphi(A)} \in f(A)$ для всех $A \in \Omega \cap K(G / D)$. Следовательно, $G / D \in \mathfrak{F}$. Поэтому $\mathfrak{F}$ является формацией. Лемма доказана.

Аналогично доказывается следующее утверждение.

Лемма 2. Пусть $f-F$-функция, $\varphi-F R$-функция $u$

$$
\mathfrak{F}=F(f, \varphi)=\left\{G \in \mathfrak{G} \mid G / G_{\varphi(A)} \text { для всех } A \in K(G)\right\}
$$

Тогда $\mathfrak{F}$ является формацией.

Определение 2. Формацию $\mathfrak{F}$ назовем $\Omega$-расслоенной, если $\mathfrak{F}=\Omega F(f, \varphi)$, где $f$ и $\varphi-$ некоторые $\Omega F$-функция и $\Omega R$-функция соответственно. Функцию $f$ будем называть $\Omega F$-спутником, а функцию $\varphi$ - направлением $\Omega$-расслоенной формации $\mathfrak{F}$. Пусть $f-F$-функция. Формацию $\mathfrak{F}=F(f, \varphi)$ назовем расслоенной формацией с направлением $\varphi$, а $f$ будем называть $F$-спутником формации $\mathfrak{F}$

Лемма 3. Пусть $\mathfrak{M}-$ формачия, $K(\mathfrak{M}) \cap \Omega=\varnothing$. Тогда $\mathfrak{M}=\Omega F(m, \varphi)$, где $m$ - $\Omega F$-функция такая, что $m\left(\Omega^{\prime}\right)=\mathfrak{M}, m(A)=\varnothing$ для всех $A \in \Omega, u \varphi-$ произвольная $F R$-функция. В частности, пустая формачия $\varnothing$ и единичная формачия (1) являются $\Omega$-расслоенными формачиями для любого непустого класса $\Omega \subseteq \mathfrak{I}$.

Доказательство. Пусть $\mathfrak{B}=\Omega F(m, \varphi), m$ и $\varphi$ определены в формулировке леммы. Покажем, что $\mathfrak{M}=\mathfrak{B}$. Если $G \in \mathfrak{M}$, то $G / O_{\Omega}(G) \in \mathfrak{M}=m\left(\Omega^{\prime}\right)$ и из $A \in \Omega \cap K(G)=\varnothing$ следует, что $G / G_{\varphi(A)} \in m(A)$. Поэтому $G \in \mathfrak{B}$ и $\mathfrak{M} \subseteq \mathfrak{B}$.

Допустим, что $\mathfrak{M} \subset \mathfrak{B}$ и $H-$ группа наименьшего порядка из $\mathfrak{B} \backslash \mathfrak{M}$. Тогда $H$ - монолитическая группа с монолитом $P=H^{\mathfrak{M}}$. Так как $H / O_{\Omega}(H) \in m\left(\Omega^{\prime}\right)=\mathfrak{M}$, то $P \subseteq O_{\Omega}(H)$. Пусть $K(P)=(B)$. Тогда $B \in K(H) \cap \Omega$ и $H / H_{\varphi(B)} \in m(B)=\varnothing$. Получили противоречие. Следовательно, $\mathfrak{M}=\mathfrak{B}$. Лемма доказана.

Определение 3. Формацию $\mathfrak{F}=\Omega F(f, \varphi)$ назовем $\Omega$-свободной или, коротко, $\Omega F r$ формацией, если $\varphi(A)=\mathfrak{G}_{\Omega^{\prime}}$ для любого $A \in \mathfrak{I}$, и будем обозначать

$\mathfrak{F}=\operatorname{Fr}(f)=\left\{G \in \mathfrak{G} \mid G / O_{\Omega}(G) \in f\left(\Omega^{\prime}\right)\right.$ и $G / O_{A^{\prime}}(G) \in f(A)$ для всех $\left.A \in \Omega \cap K(G)\right\}$,

a $f$ будем называть $\Omega F r$-спутником формации $\mathfrak{F}$. Пусть $f-F$-функция. Формацию

$$
\mathfrak{F}=\operatorname{Fr}(t)=\left\{G \in \mathfrak{G} \mid G / O_{A^{\prime}}(G) \in f(A) \text { для всех } A \in K(G)\right\}
$$

назовем свободной формацией с $F r$-спутником $f$.

Теорема 1. Пусть $\mathfrak{F}$ - непустая неединичная формация $u \Omega=K(\mathfrak{F})$. Тогда $\mathfrak{F}$ является $\Omega$-свободной формачией. 
Доказательство. Пусть $f-$ такая $\Omega F$-функция, что $f\left(\Omega^{\prime}\right)=\mathfrak{F}$,

$$
f(A)=\operatorname{form}\left(G / O_{A^{\prime}}(G) \mid G \in \mathfrak{F}\right)
$$

для всех $A \in \Omega$, и $\mathfrak{F}_{1}=\Omega \operatorname{Fr}(f)$. Покажем, что $\mathfrak{F}=\mathfrak{F}_{1}$. Пусть $H \in \mathfrak{F}$. Тогда $H / O_{\Omega}(H) \in \mathfrak{F}=f\left(\Omega^{\prime}\right)$. Кроме того, $H / O_{A^{\prime}}(H) \in\left(G / O_{A^{\prime}}(G) \mid G \in \mathfrak{F}\right) \subseteq f(A)$ для любого $A \in \Omega \cap K(H)$. Следовательно, $H \in \mathfrak{F}_{1}$ и $\mathfrak{F} \subseteq \mathfrak{F}_{1}$.

Допустим, что $\mathfrak{F} \subset \mathfrak{F}_{1}$ и $T-$ группа наименьшего порядка из $\mathfrak{F}_{1} \backslash \mathfrak{F}$. Тогда $T$ - монолитическая группа с монолитом $M=T^{\mathfrak{F}}$. Поскольку $T / O_{\Omega}(T) \in f\left(\Omega^{\prime}\right)=\mathfrak{F}$ и $\Omega=K(\mathfrak{F})$, то $T / O_{\Omega}(T)$ является $\Omega$-группой. Поскольку расширение $\Omega$-группы с помощью $\Omega$-группы является $\Omega$-группой, то $T=O_{\Omega}(T)$. Пусть $K(M)=(A)$. Тогда $A \in \Omega$ и по определению $\mathfrak{F}_{1}$ справедливо включение $T / O_{A^{\prime}}(T) \in f(A) \subset \mathfrak{F}$. Следовательно, $M \subseteq O_{A^{\prime}}(T)$. Получили противоречие. Поэтому $\mathfrak{F}=\mathfrak{F}_{1}$. Теорема доказана.

Замечание 1. Из теоремы 1 и леммы 3 следует, что каждая формация является $\Omega$-свободной для некоторого непустого класса простых групп $\Omega$. Обозначим направление свободной формации через $\varphi_{0}$.

Лемма 4. Пусть $\mathfrak{F}=\Omega F(f, \varphi)$, где $\varphi-$ произвольная $F R$-функция. Тогда справедливы следующие утверждения:

(1) $\mathfrak{F}=\Omega F(g, \varphi)$, где $g(A)=f(A) \cap \mathfrak{F}$ для любого $A \in \Omega \cup\left\{\Omega^{\prime}\right\}$;

(2) $\mathfrak{F}=\Omega F(h, \varphi)$, әде $h\left(\Omega^{\prime}\right)=\mathfrak{F} u h(A)=f(A)$ для люобого $A \in \Omega$.

Доказательство. Пусть $\mathfrak{F}_{1}=\Omega F(g, \varphi)$, где $g$ - функция, описанная в пункте 1 леммы. Так как $g(A) \subseteq f(A)$ для любого $A \in \Omega \cup\left\{\Omega^{\prime}\right\}$, то $\mathfrak{F}_{1} \subseteq \mathfrak{F}$. Пусть $G \in \mathfrak{F}$. Тогда $G / O_{\Omega}(G) \in f\left(\Omega^{\prime}\right)$ и $G / G_{\varphi(A)} \in \dot{f}(A)$ для любого $A \in \Omega \cap K(G)$. Поскольку $\mathfrak{F}$ - формация, то $\left\{G / O_{\Omega}(G), G / G_{\varphi(A)}\right\} \subseteq \mathfrak{F}$, и значит, $G / O_{\Omega}(G) \in f\left(\Omega^{\prime}\right) \cap \mathfrak{F}=g\left(\Omega^{\prime}\right)$ и $G / G_{\varphi(A)} \in f(A) \cap \mathfrak{F}=g(A)$ для любого $A \in \Omega \cap K(G)$. Следовательно, $G \in \mathfrak{F}_{1}$ и $\mathfrak{F} \subseteq \mathfrak{F}_{1}$. Таким образом, $\mathfrak{F}=\mathfrak{F}_{1}$.

Пусть $h-\Omega F$-функция из пункта 2 леммы и $\mathfrak{H}=\Omega F(h, \varphi)$. Покажем, что $\mathfrak{F}=\mathfrak{H}$. Пусть $G \in \mathfrak{F}$. Тогда $G / O_{\Omega}(G) \in \mathfrak{F}=h\left(\Omega^{\prime}\right)$ и $G / G_{\varphi(A)} \in f(A)=h(A)$ для любого $A \in \Omega \cap K(G)$. Следовательно, $G \in \mathfrak{H}$ и $\mathfrak{F} \subseteq \mathfrak{H}$.

Допустим, что $\mathfrak{F} \subset \mathfrak{H}$ и $H$ - группа наименьшего порядка из $\mathfrak{H} \backslash \mathfrak{F}$. Тогда группа $H$ монолитична с монолитом $P=H^{\mathfrak{F}}$. Из $H / O_{\Omega}(H) \in h\left(\Omega^{\prime}\right)=\mathfrak{F}$ следует, что $P \subseteq O_{\Omega}(H)$ и $H / O_{\Omega}(H) \cong(H / P) /\left(O_{\Omega}(H) / P\right)=(H / P) / O_{\Omega}(H / P) \in f\left(\Omega^{\prime}\right)$. Кроме того, для любого $A \in \Omega \cap K(H)$ справедливо включение $H / H_{\varphi(A)} \in h(A)=f(A)$. Следовательно, $H \in \mathfrak{F}$. Полученное противоречие доказывает равенство $\mathfrak{F}=\mathfrak{H}$. Лемма доказана.

Замечание 2. Пусть $A$ - простая группа и $\mathfrak{S}_{c A}-$ класс всех конечных групп, у которых каждый главный $A$-фактор централен. Если $A$ - неабелева простая группа, то $\mathfrak{S}_{c A}=\mathfrak{G}_{A^{\prime}}$. Нетрудно показать, что класс $\mathfrak{S}_{c A}$ является формацией Фиттинга и $\mathfrak{S}_{c A}$-радикал группы $G$ равен $F_{A}(G)$ (см. теоремы 5 и 7 в [13]). Обозначим $\mathfrak{S}_{c A}$ корадикал группы $G$ через $F^{A}(G)$.

Определение 4. Формация $\mathfrak{F}=\Omega F(f, \varphi)$ называется $\Omega$-композиционной или, коротко, $\Omega C$-формацией, если $\varphi(A)=\mathfrak{S}_{c A}$ для любого $A \in \mathfrak{I}$, в этом случае пишут

$$
\begin{aligned}
\mathfrak{F}= & \Omega C F(f) \\
& =\left\{G \in \mathfrak{G} \mid G / O_{\Omega}(G) \in f\left(\Omega^{\prime}\right) \text { и } G / F_{A}(G) \in f(A) \text { для любого } A \in \Omega \cap K(G)\right\},
\end{aligned}
$$


a $f$ называют $\Omega C$-спутником формации $\mathfrak{F}$ (см. $[7,8])$. В случае, когда функция $f$ является формационной, получаем определение композиционной формации

$$
\mathfrak{F}=C F(f)=\left\{G \in \mathfrak{G} \mid G / F_{A}(G) \in f(A) \text { для всех } A \in K(G)\right\}
$$

с $C$-спутником $f$.

Определение 5. Формацию $\mathfrak{F}=\Omega F(f, \varphi)$ называют $\Omega$-канонической или, коротко, $\Omega K$-формацией, если $\varphi(A)=\mathfrak{G}_{A^{\prime}} \mathfrak{G}_{A}$ для любого $A \in \mathfrak{I}$ и пишут

$$
\begin{aligned}
\mathfrak{F} & =\Omega K F(f) \\
& \left.=\left\{G \in \mathfrak{G} \mid G / O_{\Omega}(G) \in f\left(\Omega^{\prime}\right) \text { и } G /\right)_{A^{\prime}, A}(G) \in f(A) \text { для всех } A \in \Omega \cap K(G)\right\},
\end{aligned}
$$

a $f$ называют $\Omega K$-спутником формации $\mathfrak{F}$. Пусть $f-F$-функция. Формацию

$$
\mathfrak{F}=K F(t)=\left\{G \in \mathfrak{G} \mid G / O_{A^{\prime}, A}(G) \in f(A) \text { для всех } A \in K(G)\right\}
$$

назовем канонической формацией с $K$-спутником $f$.

Пример 1. Пусть $\mathfrak{F}=$ form $A$, где $A$ - простая неабелева группа. Тогда $K(\mathfrak{F})=(A)$. Пусть $\Omega=(A)$. Покажем, что $\mathfrak{F}$ не является $\Omega$-канонической формацией. Пусть

$$
\begin{aligned}
\mathfrak{F}_{1} & =\Omega K F(f) \\
& =\left\{G \in \mathfrak{G} \mid G / O_{A}(G) \in f\left(\Omega^{\prime}\right) \text { и } G / O_{A^{\prime}, A}(G) \in f(A) \text { для } A \in \Omega \cap K(G)\right\} .
\end{aligned}
$$

Если $f\left(\Omega^{\prime}\right)=\varnothing$, то условие $G / O_{A}(G)=f\left(\Omega^{\prime}\right)$ не выполняется ни для одной группы, и значит, $\mathfrak{F}_{1}=\varnothing$. Пусть $f\left(\Omega^{\prime}\right) \neq \varnothing$. Если $f(A)=\varnothing$, то условие $G / O_{A^{\prime}, A}(G) \in$ $f(A)$ для $A \in \Omega \cap K(G)$ будет выполняться лишь для $A^{\prime}$-групп. Поэтому $\mathfrak{F}_{1} \subseteq \mathfrak{G}_{A^{\prime}}$ и $\mathfrak{F}_{1} \neq \mathfrak{F}$. Пусть $f\left(\Omega^{\prime}\right) \neq \varnothing, f(A) \neq \varnothing$ и $H$ - произвольная $(A)$-группа. Тогда $H / O_{A}(H) \in f\left(\Omega^{\prime}\right)$ и $H / O_{A^{\prime}, A}(H) \in f(A)$. Следовательно, $H \in \mathfrak{F}_{1}$. Так как регулярное сплетение $A$ l $A \in \mathfrak{F}_{1} \backslash \mathfrak{F}$, то $\mathfrak{F} \neq \mathfrak{F}_{1}$. Формация $\mathfrak{F}$ является композиционной. Поэтому компіозиционная формация $\mathfrak{F}$ не обязана быть $\Omega$-канонической. Заметим, что $\mathfrak{L}=$ lform $A=\mathfrak{N}_{\pi(A)}$ form $A \neq \mathfrak{F}_{1}$. Поэтому и локальная формация $\mathfrak{L}$ не обязана быть $\Omega$-канонической.

Теорема 2. Пусть $\mathfrak{F}$ - непустая неединичная формачия $и \Omega \supseteq K(\mathfrak{F})$. Формация $\mathfrak{F}$ является $\Omega$-расслоенной с направлением $\varphi$ и $\Omega F$-спутником $f$ тогда и толъко тогда, когда $\mathfrak{F}$ - расслоенная формачия с направлением $\varphi$ и $F$-спутнихом $f_{1}$ тахим, что $f_{1}(A)=f(A)$ для любого $A \in \Omega u f_{1}(A)=\varnothing$ для любого $A \in \mathfrak{F} \backslash \Omega$.

Такие $\Omega F$-спутник $f$ и $F$-спутник $f_{1}$ формации $\mathfrak{F}$ будем называть согласованными.

Доказателъство. Докажем необходимость. Пусть формация $\mathfrak{F}$ является $\Omega$-расслоенной с направлением $\varphi$. Тогда

$$
\begin{aligned}
\mathfrak{F} & =\Omega F(f, \varphi) \\
& =\left\{G \in \mathfrak{G} \mid G / O_{\Omega}(G) \in f\left(\Omega^{\prime}\right) \text { и } G / G_{\varphi(A)} \in f(A) \text { для всех } A \in \Omega \cap K(G)\right\} .
\end{aligned}
$$

Рассмотрим $F$-функцию $f_{1}$ такую, что $f_{1}(A)=f(A)$ для любого $A \in \Omega$ и $f_{1}(A)=\varnothing$ для любого $A \in \mathfrak{I} \backslash \Omega$. Пусть

$$
\mathfrak{F}_{1}=F\left(f_{1}, \varphi\right)=\left\{H \in \mathfrak{G} \mid H / H_{\varphi(A)} \in f_{1}(A) \text { для любого } A \in K(H)\right\} .
$$


Покажем, что $\mathfrak{F}=\mathfrak{F}_{1}$. Пусть $G \in \mathfrak{F}$. Тогда $G$ является $\Omega$-группой, и значит, $G / G_{\varphi(A)} \in$ $f_{1}(A)=f(A)$ для любого $A \in K(G) \cap \Omega$. Следовательно, $G \in \mathfrak{F}_{1}$ и $\mathfrak{F} \subseteq \mathfrak{F}_{1}$.

Допустим, что $\mathfrak{F} \subset \mathfrak{F}_{1}$ и $T-$ группа минимального порядка из $\mathfrak{F}_{1} \backslash \mathfrak{F}$. Тогда $T$ - монолитическая группа с монолитом $M=T^{\mathfrak{F}}$. В силу леммы 4 можно считать, что $f\left(\Omega^{\prime}\right)=\mathfrak{F}$. Пусть $K(M)=(B)$. Поскольку $T \in \mathfrak{F}_{1}$, то $T / T_{\varphi(B)} \in f_{1}(B)$, и значит, $f_{1}(B) \neq \varnothing$. Тогда из определения $f_{1}$ следует, что $B \in \Omega$ и $M \subseteq O_{\Omega}(T)$. Поэтому $T / O_{\Omega}(T) \cong(T / M) /\left(O_{\Omega}(T) / M\right) \in \mathfrak{F}=f\left(\Omega^{\prime}\right)$ и $T / T_{\varphi(A)}=f_{1}(A)=f(A)$ для любого $A \in K(T)=\Omega \cap K(T)$. Отсюда получаем, что $T \in \mathfrak{F}$. Получили противоречие, следовательно, $\mathfrak{F}=\mathfrak{F}_{1}$.

Докажем достаточность. Пусть теперь $\mathfrak{F}=F(g, \varphi)$ - расслоенная формация с направлением $\varphi$. Рассмотрим $\Omega F$-функцию $h$ такую, что $h\left(\Omega^{\prime}\right)=\mathfrak{F}, h(A)=g(A)$ для любого $A \in \Omega$. Пусть $\mathfrak{H}=\Omega F(h, \varphi)$. Покажем, что $\mathfrak{F}=\mathfrak{H}$. Если $G \in \mathfrak{F}$, то $G / O_{\Omega}(G) \in \mathfrak{F}=h\left(\Omega^{\prime}\right)$ и для любого $A \in \Omega \cap K(G)$ справедливо включение $G / G_{\varphi(A)} \in$ $g(A)=h(A)$. Следовательно, $G \in \mathfrak{H}$ и $\mathfrak{F} \subseteq \mathfrak{H}$.

Допустим, что $\mathfrak{F} \subset \mathfrak{H}$ и $H-$ группа наименьшего порядка в $\mathfrak{H} \backslash \mathfrak{F}$. Тогда $H$ - монолитическая группа с монолитом $M=H^{\mathfrak{F}}$. Так как $H / O_{\Omega} \in h\left(\Omega^{\prime}\right)=\mathfrak{F}$, то $M \subseteq O_{\Omega}(H)$. Поскольку $H / M \in \mathfrak{F}$ и $\Omega \supseteq K(\mathfrak{F})$, то $H / M$ является $\Omega$-группой, и значит, $H-\Omega$-группа. В силу определения $\mathfrak{H}$ справедливо включение $H / H_{\varphi(A)} \in$ $h(A)$ для любого $A \in \Omega \cap K(H)=K(H)$, и из определения функции $h$ следует, что $H / H_{\varphi(A)} \in h(A)=g(A)$ для любого $A \in K(H)$. Следовательно, $H \in \mathfrak{F}$. Получили противоречие, поэтому $\mathfrak{F}=\mathfrak{H}$, и теорема доказана.

Следствие 1. Пусть $\mathfrak{F}$ - непустая неединичная формачия $u \Omega \supseteq K(\mathfrak{F})$. Формация $\mathfrak{F}$ является $\Omega$-свободной $с \Omega F r$-спутником $f$ тогда и толъко тогда, когда $\mathfrak{F}-$ свободная формация с Fr-спутником $f_{1}$, согласованным $c f$.

Следствие 2. Пустъ $\mathfrak{F}$ - непустая неединичная формация и $\Omega \supseteq K(\mathfrak{F})$. Формация $\mathfrak{F}$ является $\Omega$-композиционной $с \Omega C$-спутником $f$ тогда и только тогда, когда $\mathfrak{F}$ - композиционная формация $c$-спутником $f_{1}$, согласованным $c f$.

Следствие 3. Пусть $\mathfrak{F}$ - непустая неединичная формация $и \Omega \supseteq K(\mathfrak{F})$. Формация $\mathfrak{F}$ являетсл $\Omega$-канонической $c \Omega K$-спутником $f$ тогда и толъко тогда, когда $\mathfrak{F}$ каноническая формация с $K$-спутником $f_{1}$, согласованным $c f$.

Замечание 3. Пусть $\psi_{1}$ и $\psi_{2}$ - произвольные $\Omega F$-функции ( $F$-функции, $F R$-функции). Будем говорить, что $\psi_{1} \leqslant \psi_{2}$, если $\psi_{1}(A) \leqslant \psi_{2}(A)$ для всех $A \in \Omega \cup\left\{\Omega^{\prime}\right\}$ (для всех $A \in \mathfrak{I}$ ). Среди направлений $\varphi$ расслоенной формации с $\varphi_{0} \leqslant \varphi$ важное место занимают направления, удовлетворяющие равенству $\mathfrak{G}_{A^{\prime}} \varphi(A)=\varphi(A)$ для всех $A \in \mathfrak{I}$. Этому равенству удовлетворяют направления $\Omega$-свободной, $\Omega$-композиционной, $\Omega$-канонической формаций, а также направления вида $\varphi(A)=\mathfrak{G}_{A^{\prime}} \mathfrak{X}$ для любого $A \in \mathfrak{I}$ и любой непустой формации Фиттинга $\mathfrak{X}$.

Определение 6. Направление $\varphi$ расслоенной формации назовем правильным или, коротко, $r$-направлением, если $\varphi(A)=\mathfrak{G}_{A^{\prime}} \varphi(A)$ для любого $A \in \mathfrak{I}$.

Теорема 3. Если $\mathfrak{F}$ - расслоенная формация с $r$-направлением $\varphi$, то $\mathfrak{F}$ является $\Omega$-расслоенной формацией с $r$-направлением $\varphi$ для любого непустого класса $\Omega \subset \mathfrak{F}$.

Доказательство. Пусть $\Omega$ - непустой подкласс класса $\mathfrak{I}, \mathfrak{F}=F(f, \varphi)-$ расслоенная формация с $r$-направлением $\varphi$. Рассмотрим $\Omega F$-функцию $h$ такую, что $h\left(\Omega^{\prime}\right)=\mathfrak{F}$, 
$h(A)=f(A)$ для любого $A \in \Omega$. Пусть $\mathfrak{H}=\Omega F(h, \varphi)$. Как и при доказательстве теоремы 2 , легко проверить, что $\mathfrak{F} \subseteq \mathfrak{H}$.

Допустим, что $H \in \mathfrak{H} \backslash \mathfrak{F}$ и $H-$ группа наименьшего порядка с таким свойством. Тогда $H$ - монолитическая группа с монолитом $M=H^{\mathfrak{F}}$. Пусть $K(M)=(B)$. Как и при доказательстве теоремы $2, M \subseteq O_{\Omega}(H)$ и поэтому $B \in \Omega$. Тогда $H / H_{\varphi(B)} \in$ $h(B)=f(B)$. Пусть $A \in K(H) /(B)$. Введем обозначение $(H / M)_{\varphi(A)}=T / M$. Поскольку $T / M \in \varphi(A)$ и $M \in \mathfrak{G}_{A^{\prime}}$, то $T \in \mathfrak{G}_{A^{\prime}} \varphi(A)=\varphi(A)$. Отсюда получаем равенство $(H / M)_{\varphi(A)}=H_{\varphi(A)} / M$, и значит,

$$
H / H_{\varphi(A)} \cong(H / M) /\left(H_{\varphi(A)} / M\right)=(H / M) /(H / M)_{\varphi(A)} \in f(A) .
$$

Следовательно, $H \in \mathfrak{F}$. Пслученное противоречие доказывает равенство $\mathfrak{F}=\mathfrak{H}$. теорема доказана.

Следствие 4. Если $\mathfrak{F}$ - свободная формация, то $\mathfrak{F}$ является $\Omega$-свободной формацией для любого непустого класса $\Omega \subseteq \mathfrak{I}$.

Следствие 5 ([7]). Если $\mathfrak{F}$ - композиционная формация, то $\mathfrak{F}$ является $\Omega$-композиционной формацией для любого непустого класса $\Omega \subseteq \mathfrak{I}$.

Следствие 6. Если $\mathfrak{F}$ - каноническая формация, то $\mathfrak{F}$ является $\Omega$-канонической формацией для любого непустого класса $\Omega \subseteq \mathfrak{I}$.

Теорема 4. Пусть $\mathfrak{F} \subseteq \mathfrak{S}$. Формация $\mathfrak{F}$ является $\Omega$-канонической тогда и толъко тогда, когда $\mathfrak{F}$ является $\Omega$-композиционной.

Доказательство. Пусть $G \in \mathfrak{F}$ и $A \in K(G)$. Тогда $A \in \mathfrak{A}$. Пусть $A \cong Z_{p}$. Покажем, что $F_{Z_{p}}(G)=O_{\left(Z_{p}\right)^{\prime}, Z_{p}}(G)$. Действительно, поскольку $G \in \mathfrak{S}$, каждый главный $p d-$ фактор группы $G$ является главным $Z_{p}$-фактором группы $G$, и поэтому $F_{Z_{p}}(G)=$ $F_{p}(G)$. Так как $F_{p}(G) \in \mathfrak{G}_{p^{\prime}} \mathfrak{N}_{p}$, то $F_{Z_{p}}(G) \subseteq O_{\left(Z_{p}\right)^{\prime}, Z_{p}}(G)$. С другой стороны, $F_{p}(G)$ - наибольшая нормальная $p$-нильпотентная подгруппа группы $G$. Следовательно, $F_{Z_{p}}(G)=O_{\left(Z_{p}\right)^{\prime}, Z_{p}}(G)$. Пусть

$$
\begin{aligned}
\mathfrak{F} & =\Omega K F(f) \\
& =\left\{G \in \mathfrak{G} \mid G / O_{\Omega}(G) \in f\left(\Omega^{\prime}\right) \text { и } G / O_{A^{\prime}, A}(G) \in f(A) \text { для всех } A \in \Omega \cap K(G)\right\} .
\end{aligned}
$$

Это, в силу равенства $F_{A}(G)=O_{A^{\prime}, A}(G)$ для всех $A \in K(G)$, справедливо тогда и только тогда, когда

$$
\begin{aligned}
\mathfrak{F} & =\left\{G \in \mathfrak{G} \mid G / O_{\Omega}(G) \in f\left(\Omega^{\prime}\right) \text { и } G / F_{A}(G) \in f(A) \text { для всех } A \in \Omega \cap K(G)\right\} \\
& =\Omega C F(f) .
\end{aligned}
$$

Теорема доказана.

Следствие 7. Пусть $\mathfrak{F} \subseteq \mathfrak{S}$. Формация $\mathfrak{F}$ является канонической тогда и толъко тогда, когда $\mathfrak{F}$ является локалъной.

Доказательство проводится путем последовательного применения при $\Omega \supseteq K(\mathfrak{F})$ следствия 3 , теоремы 4 , следствия 2 и равносильности понятий композиционности и локальности для разрешимой формации. 
Пусть $\left\{f_{i} \mid i \in I\right\}-$ произвольный набор $\Omega F$-функций ( $F$-функций). Обозначим через $\bigcap_{i \in I} f_{i}$ такую $\Omega F$-функцию $(F$-функцию) $f$, что

$$
f(A)=\bigcap_{i \in I} f_{i}(A)
$$

для всех $A \in \Omega \cup\left\{\Omega^{\prime}\right\}$ (для всех $A \in \mathfrak{I}$ ).

Лемма 5. Пусть $\varphi-$ произвольная $F R$-функция, $\mathfrak{F}=\bigcap_{i \in I} \mathfrak{F}_{i}$, где $\mathfrak{F}_{i}=\Omega F\left(f_{i}, \varphi\right)$, $i \in I$. Тогда $\mathfrak{F}=\Omega F(f, \varphi)$, где $f=\bigcap_{i \in I} f_{i}$.

Доказательство. Пусть $\mathfrak{H}=\Omega F(f, \varphi)$. Покажем, что $\mathfrak{F}=\mathfrak{H}$. Пусть $G \in \mathfrak{F}$. Тогда $G \in \mathfrak{F}_{i}$, и значит, $G / O_{\Omega}(G) \in f_{i}\left(\Omega^{\prime}\right), i \in I$. Поэтому $G / O_{\Omega}(G) \in \bigcap_{i \in I} f_{i}\left(\Omega^{\prime}\right)=f\left(\Omega^{\prime}\right)$. Так как для любого $A \in \Omega \cap K(G)$ справедливы включения $G / G_{\varphi(A)} \in f_{i}(A), i \in I$, то $G / G_{\varphi(A)} \in \bigcap_{i \in I} f_{i}(A)=f(A)$. Следовательно, $G \in \mathfrak{H}$ и $\mathfrak{F} \subseteq \mathfrak{H}$.

Пусть $B \in \mathcal{H}$. Тогда $B / O_{\Omega}(B) \in f\left(\Omega^{\prime}\right)=\bigcap_{i \in I} f_{i}\left(\Omega^{\prime}\right)$ и $B / B_{\varphi(A)} \in f(A)=$ $\bigcap_{i \in I} f_{i}(A)$ для любого $A \in \Omega \cap K(B)$. Отсюда следует, что $B / O_{\Omega}(B) \in f_{i}\left(\Omega^{\prime}\right)$ и $B / B_{\varphi(A)} \in f_{i}(A)$ для любого $A \in \Omega \cap K(B), i \in I$. Поэтому $B \in \mathfrak{F}_{i}, i \in I$, и значит, $B \in \bigcap_{i \in I} \mathfrak{F}_{i}=\mathfrak{F}$. Таким образом, $\mathfrak{H} \subseteq \mathfrak{F}$. Лемма доказана.

Нетрудно проверить, что и для расслоенных формаций справедливо утверждение, аналогичное лемме 5.

Пусть $\mathfrak{X}$ - непустое множество групп. Обозначим через $\Omega F(\mathfrak{X}, \varphi)$ пересечение всех $\Omega$-расслоенных формаций с направление $\varphi$, которые содержат $\mathfrak{X}, F(\mathfrak{X}, \varphi)-$ пересечение всех расслоенных формаций с направление $\varphi$, содержащих $\mathfrak{X}$. При фиксированной $\varphi$ формации $\Omega F(\mathfrak{X}, \varphi)$ и $F(\mathfrak{X}, \varphi)$ будем также обозначать $\Omega F(\mathfrak{X})$ и $F(\mathfrak{X})$ соответственно. Далее $\Omega \operatorname{Fr}()$ и $\operatorname{Fr}(\mathfrak{X})-\Omega$-свободная и свободная формация соответственно, порожденные множеством $\mathfrak{X} ; \Omega C F(\mathfrak{X})$ и $C F(\mathfrak{X}), \Omega K F(\mathfrak{X})$ и $K F(\mathfrak{X})-$ соответственно $\Omega$-композиционная и композиционная, $\Omega$-каноническая и каноническая формации, порожденные $\mathfrak{X}$.

В силу замечания 3 можно считать, что всякое множество $\Omega F$-функций $(F$ функций) является частично упорядоченным, и имеет смысл говорить о его минимальных и максимальных элементах. $\Omega F$-функцию $(F$-функцию) $f$ назовем минимальным $\Omega F$-спутником ( $F$-спутником) $\Omega$-расслоенной (расслоенной) формации $\mathfrak{F}$ с направлением $\varphi$, если $f$ является минимальным элементом множества всех $\Omega F$ спутников ( $F$-спутников) формации $\mathfrak{F}$.

Теорема 5. Пусть $\mathfrak{X}$ - непустой класс групп. Тогда $\Omega$-расслоеннал формачия $\mathfrak{F}=\Omega F(\mathfrak{X}, \varphi)$ с направлением $\varphi$, где $\varphi_{0} \leqslant \varphi$, обладает единственным минималъным $\Omega F$-спутником $f$ таким, что

$$
f\left(\Omega^{\prime}\right)=\operatorname{form}\left(G / O_{\Omega}(G) \mid G \in \mathfrak{X}\right), \quad f(A)=\operatorname{form}\left(G / G_{\varphi(A)} \mid G \in \mathfrak{X}\right)
$$

для всех $A \in \Omega \cap K(\mathfrak{X})$ u $f(A)=\varnothing$, если $A \in \Omega \backslash K(\mathfrak{X})$.

Доказательство. При $\mathfrak{X}=(1)$ утверждение верно. Пусть $K(\mathfrak{X}) \neq \varnothing, \varphi-$ такая $F R$-функция, что $\varphi_{0} \leqslant \varphi$. Поскольку класс $\mathfrak{G}$ является $\Omega$-расслоенной формацией с направлением $\varphi$ и $\subseteq \mathfrak{G}$, то формация $\mathfrak{F}=\Omega F(\mathfrak{X}, \varphi)$ существует, и значит, множество $L$ всех $\Omega F$-спутников формации $\mathfrak{F}$ непусто. Обозначим через $f_{1}$ пересечение всех элементов из $L$. По лемме $5 \mathfrak{F}=\Omega F\left(f_{1}, \varphi\right)$ и поэтому $f_{1} \in L$. Кроме того, для любого 
элемента $h \in L$ справедливо неравенство $f_{1} \leqslant h$. Тем самым установлено, что $f_{1}-$ единственный минимальный $\Omega F$-спутник формации $\mathfrak{F}$.

Пусть $f-\Omega F$-функция, описанная в заключении теоремы. Покажем, что $f=f_{1}$. Пусть $M \in \mathfrak{X}$. Тогда $M / O_{\Omega}(M) \in f\left(\Omega^{\prime}\right)$ и из включения $K(M) \subseteq(\mathfrak{X})$ следует, что $M / M_{\varphi(A)} \in f(A)$ для всех $A \in \Omega \cap K(M)$. Это означает, что $M \in \Omega F(f, \varphi)$ и $\mathfrak{X} \subseteq \Omega F(f, \varphi)$. Следовательно, $\mathfrak{F}=\Omega F(\mathfrak{X}, \varphi) \subseteq \Omega F(f, \varphi)$.

Покажем, что $f \leqslant f_{1}$. Пусть $A \in \Omega \cap K(\mathfrak{X})$. Тогда найдется такая группа $H \in \mathfrak{F}$, что $A \in \Omega \cap K(H)$. Из $\mathfrak{F}=\Omega F\left(f_{1}, \varphi\right)$ следует $H / H_{\varphi(A)} \in f_{1}(A)$. Поэтому $f_{1}(A) \neq \varnothing$. Пусть $G \in \mathfrak{X}$. Если $A \in \Omega \cap K(G)$, то из того, что $G \in \mathfrak{F}=\Omega F\left(f_{1}, \varphi\right)$, получаем включение $G / G_{\varphi(A)} \in f_{1}(A)$.

Пусть теперь $A \in\left(\Omega \cap K(\mathfrak{X}) \backslash(\Omega \cap K(G))\right.$. Тогда $G \in \mathfrak{G}_{A^{\prime}}=\varphi_{0}(A) \subseteq \varphi(A)$, и поэтому $G / G_{\varphi(A)} \cong 1 \in f_{1}(A)$. Таким образом, $f(A)=$ form $\left(G / G_{\varphi(A)} \mid G \in \mathfrak{X}\right) \subseteq f_{1}(A)$. Кроме того, из $\mathfrak{X} \subseteq \mathfrak{F}$ следует, что $f\left(\Omega^{\prime}\right)=\operatorname{form}\left(G / O_{\Omega}(G) \mid G \in \mathfrak{X}\right) \subseteq f_{1}\left(\Omega^{\prime}\right)$. Если $A \in \Omega \backslash K(\mathfrak{X})$, то $f(A)=\varnothing \subseteq f_{1}(A)$. Следовательно, $f \leqslant f_{1}$ и $\Omega F(f, \varphi) \subseteq \Omega F\left(f_{1}, \varphi\right)$. Таким образом, $\mathfrak{F}=\Omega F(f, \varphi)$, и значит, $f \in L$. Поскольку $f_{1}$ - единственный минимальный $\Omega F$-спутник формации $\mathfrak{F}$, то $f \leqslant f_{1}$ влечет $f=f_{1}$. Теорема доказана.

Следствие 8. Пусть $f_{i}$ - минималъный $\Omega F$-спутник $\Omega$-расслоенной формации $\mathfrak{F}_{i}$ с направлением $\varphi$, где $\varphi_{0} \leqslant \varphi, i=1,2$. $\mathfrak{F}_{1} \subseteq \mathfrak{F}_{2}$ тогда и толъко тогда, когда $f_{1} \leqslant f_{2}$.

Следствие 9. Пустъ $\mathfrak{X}$ - непустой класс групп. Тогда $\Omega$-свободная формация $\mathfrak{F}=\Omega F R(\mathfrak{X})$ обладает единственным минимальным $\Omega F r$-спутником $f$ таким, ито

$$
f\left(\Omega^{\prime}\right)=\operatorname{form}\left(G / O_{\Omega}(G) \mid G \in \mathfrak{X}\right), \quad f(A)=\text { form }\left(G / O_{A^{\prime}}(G) \mid G \in \mathfrak{X}\right)
$$

для всех $A \in \Omega K(\mathfrak{X})$ u $f(A)=\varnothing$, если $A \in \Omega \backslash K(\mathfrak{X})$.

Доказательство. Поскольку $\mathfrak{F}$ является $\Omega$-расслоенной формацией с направлением $\varphi_{0}$, по теореме 5 формация $\mathfrak{F}$ обладает единственным минимальным $\Omega F r$-спутником $f$, причем $f(A)=\operatorname{form}\left(G / O_{A^{\prime}}(G) \mid G \in \mathfrak{X}\right)$ для всех $A \in \Omega \cap F(\mathfrak{X})$. Следствие доказано.

Следствие 10. Пусть $\mathfrak{X}$-непустой класс групп. Тогда свободная формачия $\mathfrak{F}=\operatorname{Fr}(\mathfrak{X})$ обладает единственным $F r$-спутником $f_{1}$ таким, что

$$
f_{1}(A)=\operatorname{form}\left(G / O_{A^{\prime}}(G) \mid G \in \mathfrak{X}\right)
$$

для всех $A \in K(\mathfrak{X}) u f_{1}(A)=\varnothing$, если $A \in \mathfrak{F} \backslash K(\mathfrak{X})$.

Доказательство. Пусть $\Omega=\mathfrak{I}$. Согласно следствию 4 формация $\mathfrak{F}$ является $\Omega$ свободной, и значит, по следствию $9, \mathfrak{F}$ обладает единственным минимальным $\Omega F r$ спутником $f$, причем $\Omega \cap K(\mathfrak{X})=K(\mathfrak{X})$. По следствию 1 формация $\mathfrak{F}$ обладает $F r$-спутником $f_{1}$, согласованным с $f$. Пусть $g_{1}$ - внутренний $F r$-спутник формации $\mathfrak{F}$ и $g$ - внутренний $\Omega F r$-спутник формации $\mathfrak{F}$, согласованный с $g_{1}$. Так как $f \leqslant g$, то и $f_{1} \leqslant g_{1}$. Следовательно, $\mathfrak{F}$ обладает единственным минимальным $F r$-спутником $f_{1}$. Следствие доказано.

Доказательства двух следующих утверждений проводятся аналогично доказательствам следствий 9 и 10 соответственно. 
Следствие 11. Пусть $\mathfrak{X}-$ непустой класс групп. Тогда $\Omega$-каноническая формачия $\mathfrak{F}=\Omega K F(\mathfrak{X})$ обладает единственным минималъным $\Omega K$-спутником $f$ таким, что

$$
f\left(\Omega^{\prime}\right)=\operatorname{form}\left(G / O_{\Omega} \mid G \in \mathfrak{X}\right), \quad f(A)=\operatorname{form}\left(G / O_{A^{\prime}, A}(G) \mid G \in \mathfrak{X}\right)
$$

для всех $A \in \Omega \cap K(\mathfrak{X})$ u $f(A)=\varnothing$, если $A \in \Omega \backslash K(\mathfrak{X})$.

Следствие 12. Пустъ $\mathfrak{X}$ - непустой класс групп. Тогда каноническая формация $\mathfrak{F}=\Omega K F(\mathfrak{X})$ обладает единственным минималъным $K$-спутником $f$ таким, что $f(A)=\operatorname{form}\left(G / O_{A^{\prime}, A}(G) \mid G \in \mathfrak{X}\right)$ для всех $A \in K(\mathfrak{X})$ u $f(A)=\varnothing$, если $A \in \mathfrak{I} \backslash K(\mathfrak{X})$.

Следствие 13 ([7, 8]). Пустъ $\mathfrak{X}$ - непустой класс групп. Тогда $\Omega$-композиционная формация $\mathfrak{F}=\Omega C F(\mathfrak{X})$ обладает единственным минимальным $\Omega C$-спутником $f$ таким, что

$$
f\left(\Omega^{\prime}\right)=\operatorname{form}\left(G / O_{\Omega} \mid G \in \mathfrak{X}\right), \quad f(A)=\operatorname{form}\left(G / F_{A}(G) \mid G \in \mathfrak{X}\right)
$$

для всех $A \in \Omega \cap K(\mathfrak{X})$ u $f(A)=\varnothing$, если $A \in \Omega \backslash K(\mathfrak{X})$.

Следствие 14. Пустъ $\mathfrak{X}-$ класс групп $u K(\mathfrak{X}) \neq \varnothing$. Тогда композиционная формация $\mathfrak{F}=C F(\mathfrak{X})$ обладает единственным минимальным $C$-спутником $f$ таким, ито $f(A)=\operatorname{form}\left(G / F_{A}(G) \mid G \in \mathfrak{X}\right)$ для всех $A \in K(\mathfrak{X})$ u $f(A)=\varnothing$, если $A \in \mathfrak{I} \backslash K(\mathfrak{X})$.

Лемма 6. Пусть $\Omega$ - непустой класс простых групп. Если формация $\mathfrak{F}$ является $\Omega$-расслоенной с $r$-направлением $\varphi$, то $\mathfrak{F}$ является $\left(Z_{p}\right)$-расслоенной формачией $c$ $r$-направлением $\varphi$ для любого $Z_{p} \in \Omega \cap K(\mathfrak{F})$.

Дохазательство. Пусть $\mathfrak{F}=\Omega F(f, \varphi)-\Omega$-расслоенная формация с $r$-направлением $\varphi$ и $Z_{p} \in \Omega \cap K(\mathfrak{F})$. Покажем, что $\mathfrak{F}$ является $\left(Z_{p}\right)$-расслоенной формацией с $r$ направлением $\varphi$. Пусть $\left(Z_{p}\right) F$-функция $f_{p}$ такова, что $f_{p}\left(Z_{p}\right)=f\left(Z_{p}\right), f_{p}\left(\left(Z_{p}\right)^{\prime}\right)=\mathfrak{F}$, и $\mathfrak{F}_{1}=\left(Z_{p}\right) F\left(f_{p}, \varphi\right)$. Покажем, что $\mathfrak{F}=\mathfrak{F}_{1}$. Пусть $G \in \mathfrak{F}$. Тогда $G / O_{p}(G) \in \mathfrak{F}=$ $f_{p}\left(\left(Z_{p}\right)^{\prime}\right)$. Если $Z_{p} \in K(G)$, то $G / G_{\varphi\left(Z_{p}\right)} \in f\left(Z_{p}\right)=f_{p}\left(Z_{p}\right)$. Следовательно, $G \in \mathfrak{F}_{1}$ и $\mathfrak{F} \subseteq \mathfrak{F}_{1}$.

Допустим, что $\mathfrak{F} \subset \mathfrak{F}_{1}$ и $H-$ группа наименьшего порядка из $\mathfrak{F}_{1} \backslash \mathfrak{F}$. Тогда группа $H$ монолитична с монолитом $P=H^{\mathfrak{F}}$. В силу леммы 4 , можно считать, что $f\left(\Omega^{\prime}\right)=\mathfrak{F}$. Тогда

$$
H / O_{\Omega}(H) \cong\left(H / O_{p}(H)\right) /\left(O_{\Omega}(H) / O_{p}(H)\right) \in f\left(\left(Z_{p}\right)^{\prime}\right)=\mathfrak{F}=\dot{f}\left(\Omega^{\prime}\right) .
$$

Пусть $A \in \Omega \cap K(H)$. Если $A \cong Z_{p}$, то $H / H_{\varphi\left(Z_{p}\right)} \in f_{p}\left(Z_{p}\right)=f\left(Z_{p}\right)$. Пусть $A$ неизоморфна $Z_{p}$. Из включения $H / O_{p}(H) \in \mathfrak{F}$ следует, что $P \subseteq O_{p}(H)$ и $P \in \mathfrak{G}_{A^{\prime}}$. Пусть $(H / P)_{\varphi(A)}=T / P$. Тогда $T \in \mathfrak{G}_{A^{\prime}} \varphi(A)=\varphi(A)$ и поэтому $T=H_{\varphi(A)}$. Отсюда получаем, что

$$
H / H_{\varphi(A)} \cong(H / P) /\left(H_{\varphi(A)} / P\right)=(H / P) /(H / P)_{\varphi(A)} \in f(A) .
$$

Следовательно, $H \in \mathfrak{F}$. Полученное противоречие доказывает равенство $\mathfrak{F}=\mathfrak{F}_{1}$. Лемма доказана.

Замечание 4. Лемма 1 не допускает обращения. Действительно, в силу примера 1 формация $\mathfrak{F}=$ form $A$, где $A-$ простая неабелева группа, не является $\Omega$-канонической формацией для $\Omega=(A)$. Однако, по лемме $3, \mathfrak{F}$ является $\left(Z_{p}\right)$-канонической для любого простого $p$, и, в частности, для любого $Z_{p} \in \Omega \cap K(\mathfrak{F})$. 
Определение 7. Правильное направление $\varphi$ расслоенной формации назовем $r n$ направлением, если $A \notin \varphi(A)$ для любой неабелевой группы $A \in \mathfrak{I}$.

Замечание 5. Направления $\Omega$-свободной и $\Omega$-композиционной формаций являются $r n$-направлениями, а направление $\Omega$-канонической формации не является $r n$ направлением. Отметим, что $r$-направления $\varphi$ расслоенной формации такие, что $\varphi(A) \subseteq \mathfrak{G}_{A^{\prime}} \mathfrak{S}$ для любой простой группы $A \in \mathfrak{I}$, также являются $r n$-направлениями.

Теорема 6. Пусть $\Omega$ - непустой класс простых групп. Формация $\mathfrak{F}$ является $\Omega$-расслоенной с rn-направлением $\varphi$ тогда и толъко тогда, когда $\mathfrak{F}$ является $\left(Z_{p}\right)$ расслоенной формацией с гп-направлением $\varphi$ для любого $Z_{p} \in \Omega \cap K(\mathfrak{F})$.

Доказательство. Необходимость следует из леммы 6.

Докажем достаточность. Пусть $\mathfrak{F}=\left(Z_{p}\right) F\left(b_{p}, \varphi\right)-\left(Z_{p}\right)$-расслоенная формация с $r n$-направлением $\varphi$ для всех $Z_{p} \in \Omega \cap K(\mathfrak{F})$. В силу леммы 4 и теоремы 5 можно считать что $b_{p}\left(\left(Z_{p}\right)^{\prime}\right)=\mathfrak{F}$ и

$$
b_{p}\left(Z_{p}\right)=\operatorname{form}\left(G / G_{\varphi\left(Z_{p}\right)} \mid G \in \mathfrak{F}\right)
$$

для любого $Z_{p} \in \Omega \cap K(\mathfrak{F})$. Пусть $b$ - такая $\Omega F$-функция, что $b\left(\Omega^{\prime}\right)=\mathfrak{F}, b\left(Z_{p}\right)=$ $b_{p}\left(Z_{p}\right)$ для любого $Z_{p} \in \Omega \cap K(\mathfrak{F}), b\left(Z_{p}\right)=\varnothing$ для всех $Z_{p} \in \Omega \backslash K(\mathfrak{F}), b(A)=\mathfrak{F}$, если $A \in \Omega \backslash \mathfrak{A}$, и $\mathfrak{B}=\Omega F(b, \varphi)$. Покажем, что $\mathfrak{F}=\mathfrak{B}$. Пусть $G \in \mathfrak{F}$. Тогда $G / O_{\Omega}(G) \in$ $\mathfrak{F}=b\left(\Omega^{\prime}\right)$. Пусть $A \in \Omega \cap K(G)$. Если $A \cong Z_{p}$, то $G / G_{\varphi\left(Z_{p}\right)} \in b_{p}\left(Z_{p}\right)=b\left(Z_{p}\right)$. Если $A \notin \mathfrak{A}$, то $G / G_{\varphi(A)} \in \mathfrak{F}=b(A)$. Следовательно, $G \in \mathfrak{B}$ и $\mathfrak{F} \subseteq \mathfrak{B}$.

Предположим, что $B \in \mathfrak{B} \backslash \mathfrak{F}$ и $B$ - группа наименьшего порядка с таким свойством. Тогда группа $B$ монолитична с монолитом $R=B^{\mathfrak{F}}$. Пусть $A \in K(R)$. Поскольку $B / O_{\Omega}(B) \in b\left(\Omega^{\prime}\right)=\mathfrak{F}$, то $R \subseteq O_{\Omega}(B)$ и $A \in \Omega$. Так как $B / B_{\varphi(A)} \in b(A)$, то $b(A) \neq \varnothing$, и значит, $A \in \Omega \cap K(\mathfrak{F})$. Пусть $A$ - неабелева группа. Так как $\varphi-r n$-направление, то $A \notin \varphi(A)$, и значит, $B_{\varphi(A)}=1$. Отсюда получаем, что

$$
B \cong B / B_{\varphi(A)} \in b(A)=\mathfrak{F},
$$

что невозможно. Поэтому $A \cong Z_{p}$. Тогда $B / B_{\varphi\left(Z_{p}\right)} \in b\left(Z_{p}\right)=b_{p}\left(Z_{p}\right)$. Кроме того,

$$
B / O_{p}(B) \cong(B / R) /\left(O_{p}(B) / R\right) \in \mathfrak{F}=b_{p}\left(\left(Z_{p}\right)^{\prime}\right) .
$$

Таким образом, $B \in\left(Z_{p}\right) F\left(b_{p}, \varphi\right)=\mathfrak{F}$. Получили противоречие. Тем самым установлено, что $\mathfrak{F}=\mathfrak{B}$. Теорема доказана.

Следствие 15. Пустъ $\Omega$ - непустой класс простых әрупп $u \Omega_{1}=\Omega \cap \mathfrak{A} \cap K(\mathfrak{F})$. Формация $\mathfrak{F}$ является $\Omega$-расслоенной с гп-направлением $\varphi$ тогда и толъко тогда, когда $\mathfrak{F}$ является $\Omega_{1}$-расслоенной формацией с $r n$-направлением $\varphi$.

Доказательство. По теореме $6, \mathfrak{F}$ является $\Omega$-расслоенной формацией с $r n$-направлением $\varphi$ тогда и только тогда, когда $\mathfrak{F}$ является $(A)$-расслоенной формацией $\mathrm{c} r n$ направлением $\varphi$ для любого $A \in \Omega \cap \mathfrak{A} \cap K(\mathfrak{F})=\Omega_{1} \cap \mathfrak{A} \cap K(\mathfrak{F})$, но это по теореме 6 выполняется тогда и только тогда, когда $\mathfrak{F}$ является $\Omega_{1}$-расслоенной с $r n$-направлением $\varphi$. Следствие доказано. 


\section{3. $\Omega$-расслоенные классы. Фиттинга групп}

В дальнейшем неоднократно будет применяться известная, по-видимому, лемма.

Лемма 7. Пустъ $N$ - нормалъная подгруппа группы G. Тогда справедливы следующие утверждения:

$$
\begin{aligned}
& \text { - } O^{\Omega}(N) \subseteq N \cap O^{\Omega}(G), \\
& \text { - если } G / N \in \mathfrak{G}_{\Omega}, \text { mo } O^{\Omega}(G)=O^{\Omega}(N), \\
& \text { - если } H \triangleleft G u G=H N, \text { mo } O^{\Omega}(H) O^{\Omega}(N) .
\end{aligned}
$$

Определение 8. Функцию $f: \Omega \cup\left\{\Omega^{\prime}\right\} \rightarrow\{$ классы Фиттинга групп $\}$, принимающую одинаковые значения на изоморфных группах из $\Omega$, назовем $\Omega$-радикальной функцией или, коротко, $\Omega R$-функцией

Функцию $g: \mathfrak{I} \rightarrow\{$ классы Фиттинга групп $\}$, принимающую одинаковые значения на изоморфных группах из $\mathfrak{I}$, назовем радикальной функцией или, коротко, $R$-функцией.

Лемма 8. Пусть $f-\Omega R$-фунжция, $\varphi-F R$-функция $u$

$$
\mathfrak{F}=\Omega R(f, \varphi)=\left\{G \in \mathfrak{G} \mid O^{\Omega}(G) \in f\left(\Omega^{\prime}\right) u G^{\varphi(A)} \in f(A) \text { для всех } A \in \Omega \cap K(G)\right\} .
$$

\section{Тогда $\mathfrak{F}$ является классом Фиттинга.}

Дохазательство. Пусть $G \in \mathfrak{F}$ и $N \triangleleft \mathfrak{G}$. Покажем, что $N \in \mathfrak{F}$. Так как по лемме 7 $O^{\Omega}(N) \subseteq O^{\Omega}(G) \in f\left(\Omega^{\prime}\right)$ и класс $f\left(\Omega^{\prime}\right)$ нормально наследствен, то $O^{\Omega}(N) \in f\left(\Omega^{\prime}\right)$. Пусть $A \in \Omega \cap K(N)$. Тогда $A \in \Omega \cap K(G)$ и по условию $S=G^{\varphi(A)} \in f(A)$. Из того, что $N S / S \triangleleft G / S \varphi$, и нормальной наследственности класса $\varphi(A)$ следует, что $N / N \cap S \in \varphi(A)$. Поэтому $T=N^{\varphi(A)} \subseteq N \cap S$. Так как $T \triangleleft S$ и класс $F(A)$ нормально наследствен, то $T \in f(A)$, и значит, $N \in \mathfrak{F}$.

Пусть $G=H K, H \triangleleft G, K \triangleleft G, H$ и. $K$ принадлежат $\mathfrak{F}$. Так как по лемме 7 $O^{\Omega}(G)=O^{\Omega}(H) O_{\Omega}(K)$ и $O^{\Omega}(H)$ и $O^{\Omega}(K)$ принадлежат классу Фиттинга $F\left(\Omega^{\prime}\right)$, то $O^{\Omega}(G) \in f\left(\Omega^{\prime}\right)$. Пусть $A \in \Omega \cap K(G)$. Покажем, что $G^{\varphi(A)} \in f(A)$. Так как $X=H^{\varphi(A)} \in f(A), Y=K^{\varphi(A)} \in f(A)$ и $f(A)$ - класс Фиттинга, то $D=X Y \in f(A)$, причем $D \triangleleft G$. Из равенства $G=H K$ следует, что $G / D=H D / D \cdot K D / D$. По модулярному тождеству Дедекинда $H D / D \cong H / H \cap D=H / X(H \cap Y)$. Поскольку $H / X \in \varphi(A)$ и $\varphi(A)-$ формация, то

$$
H / X(H \cap Y) \cong(H / X) /(X(H \cap Y) / X) \in \varphi(A),
$$

и значит, $H D / D \in \varphi(A)$. Аналогично можно показать, что $K D / D \in \varphi(A)$. Так как $\varphi(A)$ - класс Фиттинга, то $G / D=H D / D \cdot K D / D \in \varphi(A)$. Следовательно, $G^{\varphi(A)} \subseteq D$. Из включения $D \in f(A)$ получим, что $G^{\varphi(A)} \in f(A)$, и значит, $G \in \mathfrak{F}$. Поэтому $\mathfrak{F}$ является классом Фиттинга. Лемма доказана.

Аналогично доказывается следующее утверждение.

Лемма 9. Пусть $f-R$-функция, $\varphi-F R$-функция $u$

$$
\left.\left.\mathfrak{F}=R(f, \varphi)=\left\{G \in \mathfrak{G} \mid G^{\varphi(A)} \in f(A) \text { для всех } A \in K\right) G\right)\right\} .
$$

Тогда $\mathfrak{F}$ является классом Фиттинга. 
Определение 9. Класс Фиттинга $\mathfrak{F}$ назовем $\Omega$-расслоенным, если $\mathfrak{F}=\Omega R(f, \varphi)$, где $f$ и $\varphi$ - некоторые $\Omega R$-функция и $F R$-функция соответственно. Функцию $f$ будем называть $\Omega R$-спутником, а функцию $\varphi-$ направлением $\Omega$-расслоенного класса Фиттинга $\mathfrak{F}$.

Напомним, что группа $G$ называется комонолитической, если в $G$ имеется такая нормальная подгруппа $M$ (комонолит группы $G$ ), что $G / M$-простая группа и $N \subseteq M$ для любой собственной нормальной подгруппы $N$ группы $G$ (см. [6]).

Лемма 10. Пусть $\mathfrak{M}-$ класс Фиттинга $и K(\mathfrak{M}) \cap \Omega=\varnothing$. Тогда $\mathfrak{M}=\Omega R(m, \varphi)$, где $m-\Omega R$-функция такая, что $m\left(\Omega^{\prime}\right)=\mathfrak{M}, M(A)=\varnothing$ для всех $A \in \Omega, u \varphi$ - произвольная $F R$-функция. $B$ частности, классы Фиттинга $\varnothing и$ (1) являются $\Omega$-расслоенными для любого непустого класса $\Omega \subseteq \mathfrak{I}$.

Доказательство. Пусть $\mathfrak{M}_{1}=\Omega R(m, \varphi)$, где $m$ и $\varphi$ определены в лемме. Покажем, что $\mathfrak{M}=\mathfrak{M}_{1}$. Пусть $G \in \mathfrak{M}$. Тогда $O^{\Omega}(G)=G \in \mathfrak{M}=m\left(\Omega^{\prime}\right)$, и из $A \in \Omega \cap K(G)=\varnothing$ следует, что $G^{\varphi(A)} \in m(A)$. Таким образом, $G \in \mathfrak{M}_{1}$, и значит, $\mathfrak{M} \subseteq \mathfrak{M}_{1}$.

Предположим, что $\mathfrak{M} \subset \mathfrak{M}_{1}$ и $H-$ группа наименьшего порядка из $\mathfrak{M}_{1} \backslash \mathfrak{M}$. Тогда $O^{\Omega}(H) \in m\left(\Omega^{\prime}\right)=\mathfrak{M}$. Следовательно, $\mathfrak{M} \neq \varnothing$ и $|H| \neq 1$. Пусть $M_{1}$ и $M_{2}$ - собственные нормальные подгруппы группы $H$ такие, что $H=M_{1} M_{2}$. Так как $M_{i} \in \mathfrak{M}_{1}$, то в силу выбора $H$ справедливо включение $M_{i} \in \mathfrak{M}, i=1,2$, и значит, $H \in \mathfrak{M}$. Получили противоречие. Следовательно, $H$ - комонолитическая группа с комонолитом $M$. Тогда $M \in \mathfrak{M}, M=H_{\mathfrak{M}}$ и $H / M-$ простая группа. Если $H / M \notin \Omega$, то $H=O^{\Omega}(H) \in m\left(\Omega^{\prime}\right)=\mathfrak{M}$, что невозможно. Поэтому $(H / M)=(A) \subseteq \Omega$. Тогда $H^{\varphi(A)} \in m(A)=\varnothing$. Опять получаем противоречие. Таким образом, $\mathfrak{M}=\mathfrak{M}_{1}$. Лемма доказана.

Определение 10. Класс Фиттинга $\mathfrak{F}=\Omega R(f, \varphi)$ назовем $\Omega$-свободным или, коротко, $\Omega F r$-классом Фиттинга, если $\varphi(A)=\mathfrak{G}_{A^{\prime}}$ для любого $A \in \mathfrak{I}$, и будем писать

$$
\mathfrak{F}=\Omega \operatorname{Fr} R(f)=\left\{G \in \mathfrak{G} \mid O^{\Omega}(G) \in f\left(\Omega^{\prime}\right) \text { и } O^{A^{\prime}}(G) \in f(A) \text { для всех } A \in \Omega \cap K(G)\right\},
$$

a $f$ будем называть $\Omega F r$-спутником класса Фиттинга $\mathfrak{F}$. Пусть $f-R$-функция. Класс Фиттинга

$$
\mathfrak{F}=\operatorname{Fr} R(f)=\left\{G \in \mathfrak{G} \mid O^{A^{\prime}}(G) \in f(A) \text { для всех } A \in K(G)\right\}
$$

назовем свободным классом Фиттинга.

Теорема 7. Пусть $\mathfrak{F}$ - непустой неединичный класс Фиттинга $u \Omega=K(\mathfrak{F})$. Тогда $\mathfrak{F}$ является $\Omega$-свободным классом Фиттинга.

Доказательство. Пусть $\Omega R$-функция $f$ такая, что $f(A)=\operatorname{fit}\left(O^{A^{\prime}}(G) \mid G \in \mathfrak{F}\right)$ для всех $A \in \Omega, f\left(\Omega^{\prime}\right)=\mathfrak{F}$, и $\mathfrak{F}_{1}=\Omega F r R(f)$. Покажем, что $\mathfrak{F}=\mathfrak{F}_{1}$. Пусть $H \in \mathfrak{F}$. Тогда $O^{\Omega}(H) \in \mathfrak{F}=f\left(\Omega^{\prime}\right)$. Далее, $O^{A^{\prime}}(H) \in\left(O^{A^{\prime}}(G) \mid G \in \mathfrak{F}\right)$, и значит,

$$
O^{A^{\prime}}(H) \in \operatorname{fit}\left(O^{A^{\prime}}(G) \mid G \in \mathfrak{F}\right)=f(A)
$$

для любого $A \in \Omega \cap K(H)$. Следовательно, $H \in \Omega F r R(f)=\mathfrak{F}_{1}$ и $\mathfrak{F} \subset \mathfrak{F}_{1}$.

Допустим, что $\mathfrak{F} \subset \mathfrak{F}_{1}$ и $T-$ группа минимального порядка из $\mathfrak{F}_{1} \backslash \mathfrak{F}$. Как и при доказательстве леммы 10 , нетрудно проверить, что $T$ - комонолитическая группа с 
комонолитом $L=T_{\mathfrak{F}}$. Так как $O^{\Omega}(T) \in f\left(\Omega^{\prime}\right)=\mathfrak{F}$, то $O^{\Omega} \subseteq L$, и значит, $T / L \cong A \in \Omega$. Из определения $\Omega \operatorname{Fr} R(f)$ следует, что $O^{A^{\prime}}(T) \dot{\epsilon} f(A) \subseteq \mathfrak{F}$. Из комонолитичности группы $T$ следует, что $T=O^{A^{\prime}}(T)$. Поэтому $T \in \mathfrak{F}$. Получено противоречие. Следовательно, $\mathfrak{F}=\mathfrak{F}_{1}$. Теорема доказана.

Замечание 6. Из теоремы 7 и леммы 10 следует, что каждый класс Фиттинга является $\Omega$-свободным для некоторого непустого класса простых групп $\Omega$. Обозначим направление $\Omega$-свободного класса $Ф$ иттинга через $\varphi_{0}$.

Лемма 11. Пусть $\mathfrak{F}=\Omega R(f, \varphi)$, где $\varphi-$ произволъная $F R$-функция. Тогда справедливы следующие утверждения:

(1) $\mathfrak{F}=\Omega R(g, \varphi)$, где $g(A)=f(A) \mathfrak{F}$ для любого $A \in \Omega \cup\left\{\Omega^{\prime}\right\}$,

(2) $\mathfrak{F}=\Omega R(h, \varphi)$, где $f\left(\Omega^{\prime}\right)=\mathfrak{F} u h(A)=f(A)$ для всех $A \in \Omega$,

(3) если $\varphi_{0} \leqslant \varphi$ u $\Omega \subseteq K(\mathfrak{F}$, mo

$$
\mathfrak{F}=\left\{G \in \mathfrak{G} \mid O^{\Omega}(G) \in f\left(\Omega^{\prime}\right) u G^{\varphi(A)} \in f(A) \text { для всех } A \in \Omega\right\} .
$$

Доказательство. Докажем часть 1. Пусть $\mathfrak{F}_{1}=\Omega R(g, \varphi)$, где $g-\Omega R$-функция из первого утверждение леммы. Так как $g(A) \subseteq f(A)$ для любого $A \in \Omega \cup\left\{\Omega^{\prime}\right\}$, то $\mathfrak{F}_{1} \subseteq \mathfrak{F}$. Пусть $G \in \mathfrak{F}$. Тогда $O^{\Omega}(G) \in f\left(\Omega^{\prime}\right)$ и $G^{\varphi(A)} \in f(A)$ для любого $A \in$ $\Omega \cap K(G)$. Поскольку класс $\mathfrak{F}$ нормально наследствен, то $\left\{O^{\Omega}(G), G^{\varphi(A)}\right\} \subseteq \mathfrak{F}$, и значит, $O^{\Omega}(G) \in f(A) \cap \mathfrak{F}=g\left(\Omega^{\prime}\right)$ и $G^{\varphi(A)} \in f(A) \cap \mathfrak{F}=g(A)$ для любого $A \in \Omega \cap K(G)$. Следовательно, $G \in \mathfrak{F}$ и $\mathfrak{F} \subseteq \mathfrak{F}_{1}$. Таким образом, $\mathfrak{F}=\mathfrak{F}_{1}$.

Докажем часть 2 . Пусть $H-\Omega R$-функция, описанная во втором утверждении леммы, и $\mathfrak{H}=\Omega R(h, \varphi)$. Покажем, что $\mathfrak{F}=\mathfrak{H}$. Пусть $G \in \mathfrak{F}$. Тогда $O_{\Omega}(G) \in \mathfrak{F}=h\left(\Omega^{\prime}\right)$ и $G^{\varphi(A)} \in f(A)=h(A)$ для всех $A \in \Omega \cap K(G)$. Следовательно, $G \in \mathfrak{H}$ и $\mathfrak{F} \subseteq \mathfrak{H}$.

Предположим, что $\mathfrak{F} \subset \mathfrak{H}$ и $B-$ группа наименьшего порядка из $\mathfrak{H} \backslash \mathfrak{F}$. Тогда группа $B$ является комонолитической с комонолитом $M=B_{\mathfrak{F}}$. Поскольку $B \in \mathfrak{H}$, то $O^{\Omega}(B) \in h\left(\Omega^{\prime}\right)=\mathfrak{F}$, и $O^{\Omega}(B) \subseteq M$. Тогда $B / M \cong\left(B / O^{\Omega}(B)\right) /\left(M / O^{\Omega}(B)\right) \in \mathfrak{G}_{\Omega}$ и по лемме $7 O^{\Omega}(B)=O^{\Omega}(M)$. Так как $M \in \mathfrak{F}$, то $O^{\Omega}(M) \in f\left(\Omega^{\prime}\right)$, и следовательно, $O^{\Omega}(B) \in f\left(\Omega^{\prime}\right)$. Далее, $B \in \mathfrak{H}$ влечет $B^{\varphi(A)} \in h(A)=f(A)$ для любого $A \in \Omega \cap K(B)$. Таким образом, $B \in \mathfrak{F}$. Получили противоречие. Следовательно, $\mathfrak{F}=\mathfrak{H}$.

Докажем теперь часть 3 . Пусть $\varphi_{0} \leqslant \varphi, \Omega \subseteq K(\mathfrak{F})$ и $G \in \mathfrak{F}$. Если $A \in \Omega \cap K(G)$, то $G^{\varphi(A)} \in f(A)$ по определению класса $\Omega R(f, \varphi)$. Пусть $A \in \Omega \backslash K(G)$. Так как $\Omega \subseteq K(\mathfrak{F})$, то найдется такая группа $L \in \mathfrak{F}$, что $A \in K(L)$. Отсюда получаем, что $L^{\varphi(A)} \in f(A)$, и значит, $f(A) \neq \varnothing$. Поскольку $A \notin K(G)$, то $G \in \mathfrak{G}_{A^{\prime}}=\varphi_{0}(A) \subseteq \varphi(A)$ и поэтому $G^{\varphi(A)}=1 \in f(A)$. Таким образом, $G^{\varphi(A)} \in f(A)$ для всех $A \in \Omega$. Лемма доказана.

Определение 11. Класс Фиттинга $\mathfrak{F}=\Omega R(f, \varphi)$ назовем $\Omega$-композиционным или, коротко, $\Omega C$-классом Фиттинга, если $\varphi(A)=\mathfrak{S}_{c A}$ для любого $A \in \mathfrak{I}$, и пишем

$$
\mathfrak{F}=\Omega C R(f)=\left\{G \in \mathfrak{G} \mid O^{\Omega}(G) \in f\left(\Omega^{\prime}\right) \text { и } F^{A}(G) \in f(A) \text { для всех } A \in \Omega \cap K(G)\right\},
$$

a $f$ назовем $\Omega C$-спутником класса Фиттинга $\mathfrak{F}$. В случае, когда $f$ является $R$-функцией, получаем определение композиционного класса Фиттинга

$$
\mathfrak{F}=C R(f)=\left\{G \in \mathfrak{G} \mid F^{A}(G) \in f(A) \text { для всех } A \in K(G)\right\}
$$

с $C$-спутником $f$. 
Определение 12. Класс Фиттинга $\mathfrak{F}=\Omega R(f, \varphi)$ назовем $\Omega$-каноническим или, коротко, $\Omega K$-классом Фиттинга, если $\varphi(A)=\mathfrak{G}_{A} \mathfrak{G}_{A^{\prime}}$ для любого $A \in \mathfrak{I}$, и пишем

$$
\begin{aligned}
\mathfrak{F} & =\Omega K R(f) \\
& =\left\{G \in \mathfrak{G} \mid O^{\Omega}(G) \in f\left(\Omega^{\prime}\right) \text { и } O^{A, A^{\prime}}(G) \in f(A) \text { для всех } A \in \Omega \cap K(G)\right\},
\end{aligned}
$$

а $\Omega R$-функцию $f$ назовем $\Omega K$-спутником класса Фиттинга $\mathfrak{F}$. Пусть $f-R$-функция. Класс Фиттинга $\mathfrak{F}=K R(f)=\left\{G \in \mathfrak{G} \mid O^{A, A^{\prime}}(G) \in f(A)\right.$ для всех $\left.A \in K(G)\right\}$ назовем каноническим классом Фиттинга с $K$-спутником $f$.

Теорема 8. Пустъ $\mathfrak{F}$ - непустой неединичнъй класс Фиттинга $u \Omega \supseteq K(\mathfrak{F})$. Класс Фиттинга $\mathfrak{F}$ является $\Omega$-расслоенным $с$ направлением $\varphi$ и $\Omega R$-спутником $f$ тогда и толъко тогда, когда $\mathfrak{F}$ - расслоенный класс Фиттинга с направлением $\varphi$ и R-спутником $f_{1}$ таким, что $f_{1}(A)=f(A)$ для любого $A \in \Omega u f_{1}(A)=\varnothing \partial л я$ любого $A \in \mathfrak{I} \backslash \Omega$.

Такие $\Omega R$-спутник $f$ и $R$-спутник $f_{1}$ класса Фиттинга $\mathfrak{F}$ будем называть согласованными.

Доказателъство. Докажем необходимость. Пусть

$$
\begin{aligned}
\mathfrak{F} & =\Omega R(f, \varphi) \\
& =\left\{G \in \mathfrak{G} \mid O^{\Omega}(G) \in f\left(\Omega^{\prime}\right) \text { и } G^{\varphi(A)} \in f(A) \text { для всех } A \in \Omega \cap K(G)\right\} .
\end{aligned}
$$

Рассмотрим $R$-функцию $f_{1}$ такую, что $f_{1}(A)=f(A)$ для любого $A \in \Omega$ и $f_{1}(A)=\varnothing$ для любого $A \in \mathfrak{I} \backslash \Omega$. Пусть $\mathfrak{F}_{1}=R\left(f_{1}, \varphi\right)$. Покажем, что $\mathfrak{F}=\mathfrak{F}_{1}$. Пусть $G \in \mathfrak{F}$. Так как $\Omega \supseteq K(\mathfrak{F})$, то $G$ является $\Omega$-группой, и значит, $G^{\varphi(A)} \in f(A)=f_{1}(A)$ для любого $A \in K(G) \cap \Omega$. Следовательно, $G \in \mathfrak{F}_{1}$ и $\mathfrak{F} \subseteq \mathfrak{F}_{1}$.

Допустим, что $\mathfrak{F} \subset \mathfrak{F}_{1}$ и $T$ - группа минимального порядка из $\mathfrak{F}_{1} \backslash \mathfrak{F}$. Тогда $T$ - комонолитическая группа с комонолитом $M=T_{\mathfrak{F}}$. В силу леммы 11 можно считать, что $f\left(\Omega^{\prime}\right)=\mathfrak{F}$. Пусть $T / M \cong B$. Поскольку $T \in \mathfrak{F}_{1}$, то $T^{\varphi(B)} \in f_{1}(B)$, и значит, $f_{1}(B) \neq \varnothing$. Тогда из определения $f_{1}$ следует, что $B \in \Omega$ и $O^{\Omega}(T) \subseteq M$. Поэтому $O^{\Omega}(T) \in \mathfrak{F}=f\left(\Omega^{\prime}\right)$. Далее, из $M \in \mathfrak{F}$ и $B \in \Omega$ следует $K(T) \subseteq \Omega$ и $T^{\varphi(A)} \in f_{1}(A)=f(A)$ для всех $A \in K(T)$. Теперь из определения $\Omega$-расслоенного класса Фиттинга $\mathfrak{F}$ следует, что $T \in \mathfrak{F}$. Получаем противоречие. Следовательно, $\mathfrak{F}=\mathfrak{F}_{1}$.

Докажем достаточность. Пусть теперь $\mathfrak{F}=R(g, \varphi)$ - расслоенный класс Фиттинга с направлением $\varphi$. Рассмотрим $\Omega R$-функцию $h$ такую, что $h\left(\Omega^{\prime}\right)=\mathfrak{F}, h(A)=g(A)$ для любого $A \in \Omega$. Пусть $\mathfrak{H}=\Omega R(h, \varphi)$. Покажем, что $\mathfrak{F}=\mathfrak{H}$. Если $G \in \mathfrak{F}$, то $O^{\Omega}(G) \in \mathfrak{F}=h\left(\Omega^{\prime}\right)$ и $G^{\varphi(A)} \in g(A)=h(A)$ для любого $A \in \Omega \cap K(G)$. Следовательно, $G \in \mathfrak{H}$ и $\mathfrak{F} \subseteq \mathfrak{H}$.

Допустим, что $\mathfrak{F} \subset \mathfrak{H}$ и $H-$ группа наименьшего порядка из $\mathfrak{H} \backslash \mathfrak{F}$. Тогда $H$ - комонолитическая группа с комонолитом $M=H_{\mathfrak{F}}$. Так как $O^{\Omega}(H) \in h\left(\Omega^{\prime}\right)=\mathfrak{F}$, то $O^{\Omega} \subseteq M$. Из $H / M \cong\left(H / O^{\Omega}(H)\right) /\left(M / O^{\Omega}(H)\right) \in \mathfrak{G}_{\Omega}$ следует, что $K(H) \subseteq \Omega$, и поэтому $H^{\varphi(A)} \in h(A)=g(A)$ для всех $A \in \Omega \cap K(H)=K(H)$. Следовательно, $H \in \mathfrak{F}$. Получили противоречие. Таким образом, $\mathfrak{F}=\mathfrak{H}$. Теорема доказана.

Следствие 16. Пусть $\mathfrak{F}$ - непустой неединичный класс Фиттинга $u \Omega \supseteq K(\mathfrak{F})$. Класс Фиттинга $\mathfrak{F}$ являетсл $\Omega$-свободным $c \Omega F r$-спутником $f$ тогда и толъко тогда, когда $\mathfrak{F}$ - свободный класс Фиттинга с Fr-спутником $f_{1}$, согласованнъм $c f$. 
Следствие 17. Пусть $\mathfrak{F}$ - непустой неединичный класс Фиттинга $и \Omega \supseteq K(\mathfrak{F})$. Класс Фиттинга $\mathfrak{F}$ является $\Omega$-композиционным $с \Omega C$-спутником $f$ тогда и только тогда, когда $\mathfrak{F}$ - композиционный класс Фиттинга с $C$-спутником $f_{1}$, согласованным $c f$.

Следствие 18. Пустъ $\mathfrak{F}$ - непустой неединичный класс Фиттинга $u \Omega \supseteq K(\mathfrak{F})$. Класс Фиттинга $\mathfrak{F}$ является $\Omega$-каноническим $c \Omega K$-спутником $f$ тогда и толъко тогда, когда $\mathfrak{F}$ - каноническим класс Фиттинга с $K$-спутником $f_{1}$, согласованнъм $c f$.

Определение 13. Направление $\varphi$ расслоенного класса Фиттинга назовем правильным или, коротко, $r$-направлением, если $\varphi(A)=\varphi(A) \mathfrak{G}_{A^{\prime}}$ для любого $A \in \mathfrak{I}$.

Теорема 9. Если $\mathfrak{F}$ - расслоенный класс Фиттинга $c r$-направлением $\varphi$, то $\mathfrak{F}$ является $\Omega$-расслоенным классом Фиттинга $c$ г-направлением $\varphi$ для любого непустого класса $\Omega \subseteq \mathfrak{I}$.

Доказательство. Пусть $\Omega$ - непустой подкласс класса $\mathfrak{I}, \mathfrak{F}=R(f, \varphi)$ - расслоенный класс Фиттинга с $r$-направлением $\varphi$. Рассмотрим $\Omega R$-функцию $h$ такую, что $h\left(\Omega^{\prime}\right)=\mathfrak{F}, h(A)=f(A)$ для любого $A \in \Omega$. Пусть $\mathfrak{H}=\Omega R(h, \varphi)$. Как и при доказательстве теоремы 8 , легко проверить, что $\mathfrak{F} \subseteq \mathfrak{H}$.

Допустим, что $H \in \mathfrak{H} \backslash \mathfrak{F}$ и $H$ - группа наименьшего порядка с таким свойством. Тогда $H$ - комонолитическая группа с монолитом $M=H_{\mathfrak{F}}$. Пусть $K(M)=(B)$. Как и в доказательстве теоремы $8, O^{\Omega}(H) \subseteq M$ и $H / M \cong\left(H / O^{\Omega}(H)\right) /\left(M / O^{\Omega}(H)\right) \in \mathfrak{G}_{\Omega}$. Поэтому $B \in \Omega$ и $H^{\varphi(B)} \in h(B)=f(B)$. Пусть $A \in K(H) \backslash(B)$. Тогда $A \in K(M)$ и $H / M \in \mathfrak{G}_{A^{\prime}}$. Так как $H / M \cong\left(H / M^{\varphi(A)}\right) /\left(M / M^{\varphi(A)}\right)$, то $H / M^{\varphi(A)} \in \varphi(A) \mathfrak{G}_{A^{\prime}}=\varphi(A)$ и $H^{\varphi(A)} \subseteq M^{\varphi(A)}$. Из $M \in \mathfrak{F}$ получаем, что $H^{\varphi(A)} \in f(A)$. Таким образом, $H \in \mathfrak{F}$. Полученное противоречие доказывает равенство $\mathfrak{F}=\mathfrak{H}$. Теорема доказана.

Следствие 19. Если $\mathfrak{F}$ - свободный класс Фиттинга, то $\mathfrak{F}$ является $\Omega$-свободным классом Фиттинга для любого непустого класса $\Omega \subseteq \mathfrak{I}$.

Следствие 20. Если $\mathfrak{F}$ - композиционный класс Фиттинга, то $\mathfrak{F}$ является $\Omega$ композиционнъм классом Фиттинга для любого непустого класса $\Omega \subseteq \mathfrak{I}$.

Следствие 21. Если $\mathfrak{F}$ - канонический класс Фиттинга, то $\mathfrak{F}$ является $\Omega$-каноническим классом Фиттинга для любого непустого класса $\Omega \subseteq \mathfrak{I}$.

Пусть $\left\{f_{i} \mid i \in I\right\}-$ произвольный набор $\Omega R$-функций ( $R$-функций). Обозначим через $\bigcap_{i \in I} f_{i}$ такую $\Omega R$-функцию $(R$-функцию) $f$, что

$$
f(A)=\bigcap_{i \in I} f_{i}(A)
$$

для всех $A \in \Omega \cup\left\{\Omega^{\prime}\right\} \quad(A \in \mathfrak{I})$.

Лемма 12. Пусть $\varphi-$ произвольная $\Omega R$-функция,

$$
\mathfrak{F}=\bigcap_{i \in I} \mathfrak{F}_{i}
$$

əде $\mathfrak{F}_{i}=\Omega R\left(f_{i}, \varphi\right)\left(\mathfrak{F}_{i}=R\left(f_{i}, \varphi\right)\right), i \in I$. Tогда $\mathfrak{F}=\Omega R(f, \varphi)(\mathfrak{F}=R(f, \varphi))$, где

$$
f=\bigcap_{i \in I} f_{i}
$$


Доказательство проводится аналогично доказательству леммы 5.

Пусть $\mathfrak{X}$ - непустое множество групп. Обозначим через $\Omega R(\mathfrak{X}, \varphi)$ пересечение всех $\Omega$-расслоенных классов Фиттинга с направлением $\varphi$, которые содержат $\mathfrak{X}$, и через $R(\mathfrak{X}, \varphi)$ пересечение всех расслоенных классов Фиттинга с направлением $\varphi$, содержащих $\mathfrak{X}$. При фиксированном $\varphi$ классы Фиттинга $\Omega R(\mathfrak{X}, \varphi)$ и $R(\mathfrak{X}, \varphi)$ будем также коротко обозначать $\Omega R(\mathfrak{X})$ и $R(\mathfrak{X})$ соответственно. Далее $\Omega F r(\mathfrak{X})$ и $\operatorname{Fr} R(\mathfrak{X})-$ $\Omega$-свободный и свободный классы Фиттинга соответственно, порожденные множеством $\mathfrak{X} ; \Omega C R(\mathfrak{X})$ и $C R(\mathfrak{X}), \Omega K R(\mathfrak{X})$ и $K R(\mathfrak{X})$ - соответственно $\Omega$-композиционный и композиционный, $\Omega$-канонический и канонический классы Фиттинга, порожденные классом $\mathfrak{X}$.

Пусть $f_{1}$ и $f_{2}$ - произвольные $\Omega R$-функции ( $R$-функции). Будем говорить, что $f_{1} \leqslant f_{2}$, если $f_{1}(A) \subseteq f_{2}(A)$ для всех $A \in \Omega \cup\left\{\Omega^{\prime}\right\}(A \in \mathfrak{I})$. Пусть $\left\{f_{i} \mid i \in I\right\}$ - совокупность всех $\Omega R$-спутников $\Omega$-расслоенного класса Фиттинга $\mathfrak{F}$ с фиксированным направлением $\varphi$. Назовем $\Omega R$-функцию $f$ минимальным $\Omega R$-спутником $\Omega$-расслоенного класса Фиттинга $\mathfrak{F}$, если $f$ является минимальным элементом множества $\left\{f_{i} \mid i \in I\right\}$. Аналогично определяется минимальный $R$-спутник расслоенного класса Фиттинга.

Теорема 10. Пустъ $\mathfrak{X}$-непустой класс әрупп. Тогда $\Omega$-расслоенный класс Фиттинга $\mathfrak{F}=\Omega R(\mathfrak{X}, \varphi)$ с направлением $\varphi$, где $\varphi_{0} \leqslant \varphi$, обладает единственным минималъным $\Omega R$-спутником $f$ таким, что

$$
f\left(\Omega^{\prime}\right)=\operatorname{fit}\left(O^{\Omega}(G) \mid G \in \mathfrak{X}\right), \quad f(A)=\operatorname{fit}\left(G^{\varphi(A)} \mid G \in \mathfrak{X}\right)
$$

для всех $A \in \Omega \cap K(\mathfrak{X})$ u $f(A)=\varnothing$, если $A \in \Omega \backslash K(\mathfrak{X})$.

Доказателъство. Так как $\mathfrak{G}$ является $\Omega$-расслоенным классом Фиттинга с направлением $\varphi$, где $\varphi_{0} \leqslant \varphi$, и $\mathfrak{X} \subseteq \mathfrak{G}$, то класс Фиттинга $\mathfrak{F}=\Omega R(\mathfrak{X}, \varphi)$ существует, и значит, множество $L$ всех $\Omega R$-спутников класса Фиттинга $\mathfrak{F}$ непусто. Обозначим $f_{1}$ пересечение всех элементов из $L$. По лемме $12 \mathfrak{F}=\Omega R\left(f_{1}, \varphi\right)$ и $f_{1}$ - единственный минимальный $\Omega R$-спутник класса Фиттинга $\mathfrak{F}$.

Пусть $f-\Omega R$-функция, описанная в теореме. Покажем, что $f=f_{1}$. Пусть $M \in \mathfrak{X}$. Тогда $O^{\Omega}(M) \in f\left(\Omega^{\prime}\right)$ и из $K(M) \subseteq K(\mathfrak{X})$ следует, что $M^{\varphi(A)} \in f(A)$ для всех $A \in \Omega \cap K(M)$. Это означает, что $M \in \Omega R(f, \varphi)$ и $\mathfrak{X} \subseteq \Omega R(f, \varphi)$. Следовательно, $\mathfrak{F}=\Omega R(\mathfrak{X}, \varphi) \subseteq \Omega R(f, \varphi)$

Покажем, что $\Omega R(f, \varphi) \subseteq \mathfrak{F}$. Пусть $A \in \Omega \cap K(\mathfrak{X})$. Тогда найдется такая группа $H \in \mathfrak{F}$, что $A \in \Omega \cap K(H)$. Из равенства $\mathfrak{F}=\Omega R\left(f_{1}, \varphi\right)$ следует, что $H^{\varphi(A)} \in f_{1}(A)$. Поэтому $f_{1}(A) \neq \varnothing$. Пусть $G \in \mathfrak{X}$. Если $A \in \Omega \cap K(G)$, то из $G \in \mathfrak{F}=\Omega R\left(f_{1}, \varphi\right)$ получаем, что $G^{\varphi(A)} \in f_{1}(A)$. Пусть теперь $A \in(\Omega \cap K(\mathfrak{X})) \backslash(\Omega \cap K(G))$. Тогда $G \in \mathfrak{G}_{A^{\prime}}=\varphi_{0}(A) \subseteq \varphi(A)$ и поэтому $G^{\varphi(A)}=1 \in f_{1}(A)$. Таким образом,

$$
f(A)=\operatorname{fit}\left(G^{\varphi(A)} \mid G \in \mathfrak{X}\right) \subseteq f_{1}(A) .
$$

Кроме того, из $\mathfrak{X} \subseteq \mathfrak{F}$ следует, что $f\left(\Omega^{\prime}\right)=\operatorname{fit}\left(O^{\Omega}(G) \mid G \in \mathfrak{X}\right) \subseteq f_{1}\left(\Omega^{\prime}\right)$. Если $A \in \Omega \backslash K(\mathfrak{X})$, то $f(A)=\varnothing \subseteq f_{1}(A)$. Следовательно, $f \leqslant f_{1}$ и $\Omega R(f, \varphi) \subseteq \Omega R\left(f_{1}, \varphi\right)$. Тем самым установлено, что $\mathfrak{F}=\Omega R(f, \varphi)$, и значит, $f \in L$. Поскольку $f_{1}$ - единственный минимальный $\Omega R$-спутник класса Фиттинга $\mathfrak{F}$, то $f \leqslant f_{1}$ влечет $f=f_{1}$. Теорема доказана. 
Следствие 22. Пусть $f_{i}-$ минималъный $\Omega R$-спутник $\Omega$-расслоенного класса Фиттинга $\mathfrak{F}_{i}$ с направлением $\varphi$, где $\varphi_{0} \leqslant \varphi, i=1,2$. Включение $\mathfrak{F}_{1} \subseteq \mathfrak{F}_{2}$ справедливо тогда и только тогда, когда $f_{1} \leqslant f_{2}$.

Следствие 23. Пусть $\mathfrak{X}$ - непустой класс групп. Тогда $\Omega$-свободный класс Фиттинга $\mathfrak{F}=\Omega F r R(\mathfrak{X})$ обладает единственным минимальным $\Omega F r$-спутником $f$ таким, что

$$
f\left(\Omega^{\prime}\right)=\operatorname{fit}\left(O^{\Omega}(G) \mid G \in \mathfrak{X}\right), \quad f(A)=\operatorname{fit}\left(O^{A^{\prime}}(G) \mid G \in \mathfrak{X}\right)
$$

для всех $A \in \Omega \cap K(\mathfrak{X})$ u $f(A)=\varnothing$, если $A \in \Omega \backslash K(\mathfrak{X})$.

Следствие 24. Пустъ $\mathfrak{X}$ - непустой класс групп. Тогда свободный класс Фиттинга $\mathfrak{F}=\operatorname{Fr} R(\mathfrak{X})$ обладает единственным минимальным $\mathrm{Fr}$-спутником $f_{1}$ таким, что $f_{1}(A)=\operatorname{fit}\left(O^{A^{\prime}}(G) \mid G \in \mathfrak{X}\right)$ для всех $A \in K(\mathfrak{X}) u f_{1}(A)=\varnothing$, если $A \in \mathfrak{I} \backslash K(\mathfrak{X})$.

Доказателъство. Пусть $\Omega=\mathfrak{I}$. Согласно следствию 19 класс Фиттинга $\mathfrak{F}$ является $\Omega$-свободным, и значит, по следствию 23 класс $\mathfrak{F}$ обладает единственным минимальным $\Omega F r$-спутником $f$, причем $\Omega \cap K(\mathfrak{X})=K(\mathfrak{X})$. По следствию 16 класс Фиттинга $\mathfrak{F}$ обладает $F r$-спутником $f_{1}$, согласованным с $f$. Пусть $g_{1}$ - внутренний $F r$-спутник класса Фиттинга $\mathfrak{F}$ и $g$ - внутренний $\Omega F r$-спутник класса Фиттинга $\mathfrak{F}$, согласованный с $g_{1}$. Так как $f \leqslant g$, то и $f_{1} \leqslant g_{1}$. Следовательно, $\mathfrak{F}$ обладает единственным минимальным $F r$-спутником $f_{1}$. Следствие доказано.

Следствие 25. Пусть $\mathfrak{X}$ - непустой класс групп. Тогда $\Omega$-канонический класс Фиттинга $\mathfrak{F}=\Omega K R(\mathfrak{X})$ обладает единственным минималъным $\Omega K$-спутником $f$ таким, что

$$
f\left(\Omega^{\prime}\right)=\operatorname{fit}\left(O^{\Omega}(G) \mid G \in \mathfrak{X}\right), \quad f(A)=\operatorname{fit}\left(O^{A, A^{\prime}}(G) \mid G \in \mathfrak{X}\right)
$$

для всех $A \in \Omega \cap K(\mathfrak{X})$ u $f(A)=\varnothing$, если $A \in \Omega \backslash K(\mathfrak{X})$.

Следствие 26. Пустъ $\mathfrak{X}$ - непустой класс групп. Тогда канонический класс Фиттинга $\mathfrak{F}=K R(\mathfrak{X})$ обладает единственным минималъным $K$-спутником $f$ таким, что $f(A)=\operatorname{fit}\left(O^{A, A^{\prime}}(G) \mid G \in \mathfrak{X}\right)$ для всех $A \in K(\mathfrak{X})$ u $f(A)=\varnothing$, если $A \in \mathfrak{I} \backslash K(\mathfrak{X})$.

Следствие 27. Пустъ $\mathfrak{X}$ - непустой класс әрупп. Тогда $\Omega$-композиционный класс Фиттинга $\mathfrak{F}=\Omega C R(\mathfrak{X})$ обладает единственнъм минималънъм $\Omega C$-спутником $f$ такuм, что

$$
f\left(\Omega^{\prime}\right)=\operatorname{fit}\left(O^{\Omega}(G) \mid G \in \mathfrak{X}\right), \quad f(A)=\operatorname{fit}\left(F^{A}(G) \mid G \in \mathfrak{X}\right)
$$

для всех $A \in \Omega \cap K(\mathfrak{X})$ u $f(A)=\varnothing$, если $A \in \Omega \backslash K(\mathfrak{X})$.

Следствие 28. Пусть $\mathfrak{X}$ - непустой класс групп. Тогда композиционнъй класс Фиттинга $\mathfrak{F}=C R(\mathfrak{X})$ обладает единственным минимальным $C$-спутником $f$ maxим, ито $f(A)=\operatorname{fit}\left(F^{A}(G) \mid G \in \mathfrak{X}\right)$ для всех $A \in K(\mathfrak{X})$ и $f(A)=\varnothing$, если $A \in \mathfrak{I} \backslash K(\mathfrak{X})$.

Лемма 13. Пусть $\Omega$ - непустой класс простых әрупп. Если класс Фиттинга $\mathfrak{F}$ является $\Omega$-расслоенным с $г$-направлением $\varphi$, то $\mathfrak{F}$ является $\left(Z_{p}\right)$-расслоенным $c$ $r$-направлением $\varphi$ для любого $Z_{p} \in \Omega \cap K(\mathfrak{F})$. 
Доказательство. Пусть $\mathfrak{F}=\Omega R(f, \varphi)-\Omega$-расслоенный класс Фиттинга с $r$-направлением $\varphi$ и $Z_{p} \in \Omega \cap K(\mathfrak{F})$. Покажем, что $\mathfrak{F}$ является $\left(Z_{p}\right)$-расслоенным классом Фиттинга с направлением $\varphi$. Пусть $f_{p}$ - такая $\left(Z_{p}\right) R$-функция, что $f_{p}\left(Z_{p}\right)=f\left(Z_{p}\right)$, $f_{p}\left(\left(Z_{p}\right)^{\prime}\right)=\mathfrak{F}$, и пусть $\mathfrak{F}_{1}=\left(Z_{p}\right) R\left(f_{p}, \varphi\right)$. Покажем, что $\mathfrak{F}=\mathfrak{F}_{1}$. Пусть $G \in \mathfrak{F}$. Тогда $O^{p}(G) \in \mathfrak{F}=f_{p}\left(\left(Z_{p}\right)^{\prime}\right)$. Если $Z_{p} \in K(G)$, то $G^{\varphi\left(Z_{p}\right)} \in f\left(Z_{p}\right)=f_{p}\left(Z_{p}\right)$. Следовательно, $G \in \mathfrak{F}_{1}$ и $\mathfrak{F} \subseteq \mathfrak{F}_{1}$.

Допустим, что $\mathfrak{F} \subset \mathfrak{F}_{1}$ и $H-$ группа наименьшего порядка из $\mathfrak{F}_{1} \backslash \mathfrak{F}$. Тогда группа $H$ комонолитична с монолитом $M=H_{\mathfrak{F}}$. Ввиду леммы 11 можно считать, что $f\left(\Omega^{\prime}\right)=\mathfrak{F}$. Тогда из включения $Z_{p} \in \Omega$ следует, что $O^{\Omega}(H) \subseteq O^{p}(H)$, и значит, $O^{\Omega}(H) \in f\left(\left(Z_{p}\right)^{\prime}\right)=\mathfrak{F}=f\left(\Omega^{\prime}\right)$. Пусть $A \in \Omega \cap K(H)$. Если $A \cong Z_{p}$, то

$$
H^{\varphi\left(Z_{p}\right)} \in f_{p}\left(Z_{p}\right)=f\left(Z_{p}\right) .
$$

Пусть $A$ неизоморфна $Z_{p}$. Из $O^{p}(H) \in \mathfrak{F}$ следует, что $O^{p}(H) \subseteq M$. Тогда

$$
H / M \cong\left(H / O^{p}(H)\right) /\left(M / O^{p}(H)\right) \in \mathfrak{G}_{A^{\prime}} .
$$

Так как $H / M \cong\left(H / M^{\varphi(A)}\right) /\left(M / M^{\varphi(A)}\right)$, то $\left(H / M^{\varphi(A)}\right) \in \varphi(A) \mathfrak{G}_{A^{\prime}}=\varphi(A)$ и $H^{\varphi(A)} \subseteq M^{\varphi(A)} \in f(A)$. Следовательно, $H \in \mathfrak{F}$. Полученное противоречие доказывает равенство $\mathfrak{F}=\mathfrak{F}_{1}$. Лемма доказана.

Определение 14. Правильное направление $\varphi$ расслоенного класса Фиттинга назовем $r n$-направлением, если $A \notin \varphi(A)$ для любой неабелевой простой группы $A \in \mathfrak{I}$.

Замечание 7. Как и для формаций, направления $\Omega$-свободного и $\Omega$-композиционного классов $\Phi$ иттинга являются $r n$-направлениями, а направление $\Omega$-канонического класса Фиттинга не является $r n$-направлением. Отметим, что $r$-направления $\varphi$ расслоенного класса Фиттинга такие, что $\varphi(A) \subseteq \mathfrak{S G}_{A^{\prime}}$ для любой простой группы $A \in \mathfrak{I}$, также являются $r n$-направлениями.

Теорема 11. Пусть $\Omega$ - непустой класс простых групn. Класс Фиттинга $\mathfrak{F}$ является $\Omega$-расслоенным с $r$-направлением $\varphi$ тогда и толъко тогда, когда $\mathfrak{F}$ является $\left(Z_{p}\right)$-расслоенным классом Фиттинга $c$ гn-направлением $\varphi$ для любого $Z_{p} \in \Omega \cap K(\mathfrak{F})$.

Доказательство. Необходимость следует из леммы 13. Докажем достаточность. Пусть $\mathfrak{F}=\left(Z_{p}\right) R\left(b_{p}, \varphi\right)-\left(Z_{p}\right)$-расслоенный класс Фиттинга с $r n$-направлением $\varphi$ для всех $Z_{p} \in \Omega \cap K(\mathfrak{F})$. В силу леммы 11 и теоремы 10 можно считать, что $b_{p}\left(\left(Z_{p}\right)^{\prime}\right)=\mathfrak{F}$ и $b_{p}\left(Z_{p}\right)=\operatorname{fit}\left(G^{\varphi\left(Z_{p}\right)} \mid G \in \mathfrak{F}\right)$ для любого $Z_{p} \in \Omega \cap K(\mathfrak{F})$. Пусть $b$ - такая $\Omega R$-функция, что $b\left(\Omega^{\prime}\right)=\mathfrak{F}$ и $b\left(Z_{p}\right)=b_{p}\left(Z_{p}\right)$ для любого $Z_{p} \in \Omega \cap K(\mathfrak{F})$, $b\left(Z_{p}\right)=\varnothing$ для всех $Z_{p} \in \Omega \backslash K(\mathfrak{F}), b(A)=\mathfrak{F}$, если $A \in \Omega \backslash \mathfrak{A}$, и $\mathfrak{B}=\Omega R(b, \varphi)$. Покажем, что $\mathfrak{F}=\mathfrak{B}$. Пусть $G \in \mathfrak{F}$. Тогда $O^{\Omega}(G) \in \mathfrak{F}=b\left(\Omega^{\prime}\right)$. Пусть $A \in \Omega \cap K(G)$. Если $A \cong Z_{p}$, то $G^{\varphi\left(Z_{p}\right)} \in b_{p}\left(Z_{p}\right)=b\left(Z_{p}\right)$. Если $A \in \mathfrak{A}$, то $G^{\varphi(A)} \in \mathfrak{F}=b(A)$. Следовательно, $G \in \mathfrak{B}$ и $\mathfrak{F} \subseteq \mathfrak{B}$.

Предположим, что $B \in \mathfrak{B} \backslash \mathfrak{F}$ и $B$ - группа наименьшего порядка с таким свойством. Тогда группа $B$ комонолитична с монолитом $M=B_{\mathfrak{F}}$. Пусть $A \in K(B / M)$. Поскольку $O^{\Omega}(B) \in b\left(\Omega^{\prime}\right)=\mathfrak{F}$, то $O^{\Omega}(B) \subseteq M$. Тогда

$$
B / M \cong\left(B / O^{\Omega}(B)\right) /\left(M / O^{\Omega}(B)\right) \in \mathfrak{G}_{\Omega},
$$


и значит, $A \in \Omega$. Пусть $A \notin \mathfrak{A}$. Тогда $A \notin \varphi(A)$. Если $B^{\varphi(A)} \subset B$, то

$$
B / M \cong\left(B / B^{\varphi(A)}\right) /\left(M / B^{\varphi(A)}\right) \in \varphi(A),
$$

что невозможно. Следовательно, $B=B^{\varphi(A)} \in b(A)=\mathfrak{F}$. Получаем противоречие. Таким образом, $A \cong Z_{p}$. Если $Z_{p} \notin K(\mathfrak{F})$, то $b\left(Z_{p}\right)=\varnothing$, что в силу $B^{\varphi\left(Z_{p}\right)} \in b\left(Z_{p}\right)$ невозможно. Следовательно, $Z_{p} \in K(\mathfrak{F})$ и $B^{\varphi\left(Z_{p}\right)} \in b\left(Z_{p}\right)=b_{p}\left(Z_{p}\right)$. Кроме того, из $B / M \in \mathfrak{A}_{p}$ следует, что $O^{p}(B) \subseteq M$ и поэтому $O^{p}(B) \in \mathfrak{F}=b_{p}\left(\left(Z_{p}\right)^{\prime}\right)$. Таким образом, $B \in\left(Z_{p}\right) R\left(b_{p}, \varphi\right)=\mathfrak{F}$. Получаем противоречие. Тем самым установлено, что $\mathfrak{F}=\mathfrak{B}$. Теорема доказана.

Следствие 29. Пусть $\Omega$ - непустой класс простых әрупп $u \Omega_{1}=\Omega \cap \mathfrak{A} \cap K(\mathfrak{F})$. Класс Фиттинга $\mathfrak{F}$ является $\Omega$-расслоенным классом Фиттинга с гп-направлением $\varphi$ тогда и толъко тогда, когда $\mathfrak{F}$ является $\Omega_{1}$-расслоенным классом Фиттинга $c$ гп-направлением $\varphi$.

Доказательство проводится аналогично доказательству следствия 15.

\section{Список литературы}

1. Gaschütz W., Theorie der endlichen auflösdaren Gruppen. Math. Z. (1963) 80, №4, 300-305.

2. Шеметков Л. А., Ступенчатые формации групп. Матем. сб. (1974) 94, №4, 628-648.

3. Doerk K., Hawkes T., Finite soluble groups. Gruyter, Berlin, 1992.

4. Hartley B., On Fischer's analysation of formation theory. Proc. London Math. Soc. (1969) 3, №9, 193-207.

5. Скиба А. Н., Шеметков Л. А., О частично локальных формациях. Докл. АН Беларуси (1995) 39, №3, 123-143.

6. Скиба А. Н., Шеметков Л. А., Кратно $\omega$-локальные формации и классы Фиттинга конечных групп. Матем. труды (1999) 2, №1, 1-34.

7. Ведерников В. А., Коптюх Д. Г., Частично композиционные формации групп. Препринт №2. БГПУ, Брянск, 1999, 1-28.

8. Скиба А. Н., Шеметков Л. А., Частично композиционные формации конечных групп. Докл. АН Беларуси (1999) 43, №4, 5-8.

9. Шеметков Л. А., Формации конечнъх групп. Наука, Москва, 1978.

10. Шеметков Л. А., Скиба А. Н., Формачии алгебрачческих систем. Наука, Москва, 1989.

11. Скиба А. Н., Шеметков Л. А., О минимальном композиционном экране композиционной формации. Вопросы алгебры (1992) 7, 39-43.

12. Ведерников В. А., Прямые произведения и формации конечных групп. Алгебра и логика (1990) 29, №10, 523-548.

13. Ведерников В. А., О некоторых классах конечных групп. Докл. АН БССР (1988) 32 , №10, 872-875.

Статья поступила 23.03.2000. 